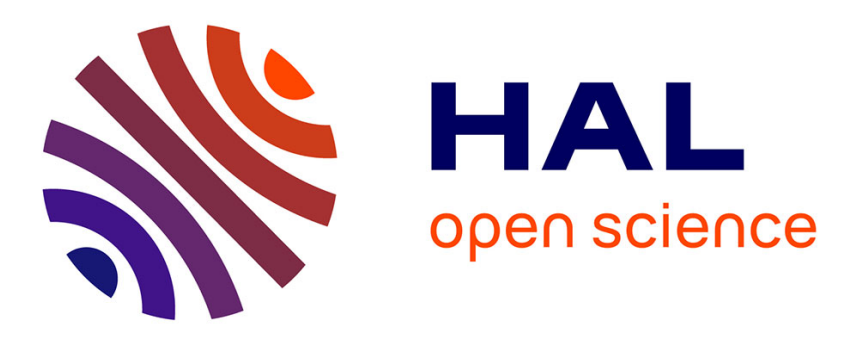

\title{
Nonlinear control of unsteady finite-amplitude perturbations in the Blasius boundary-layer flow
}

Stefania Cherubini, Jean-Christophe Robinet, Pietro de Palma

\section{To cite this version:}

Stefania Cherubini, Jean-Christophe Robinet, Pietro de Palma. Nonlinear control of unsteady finiteamplitude perturbations in the Blasius boundary-layer flow. Journal of Fluid Mechanics, 2013, 737, pp.440-465. 10.1017/jfm.2013.576 . hal-01088714

\section{HAL Id: hal-01088714 https://hal.science/hal-01088714}

Submitted on 28 Nov 2014

HAL is a multi-disciplinary open access archive for the deposit and dissemination of scientific research documents, whether they are published or not. The documents may come from teaching and research institutions in France or abroad, or from public or private research centers.
L'archive ouverte pluridisciplinaire HAL, est destinée au dépôt et à la diffusion de documents scientifiques de niveau recherche, publiés ou non, émanant des établissements d'enseignement et de recherche français ou étrangers, des laboratoires publics ou privés. 


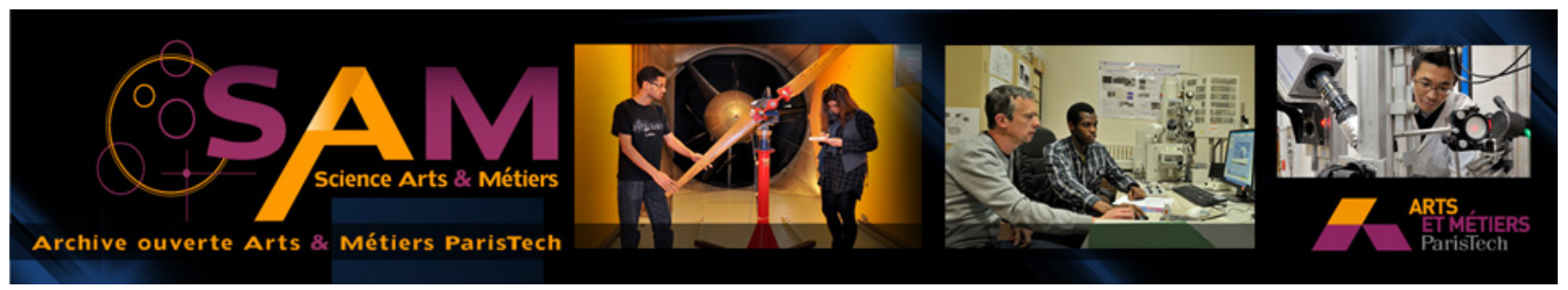

Science Arts \& Métiers (SAM)

is an open access repository that collects the work of Arts et Métiers ParisTech researchers and makes it freely available over the web where possible.

This is an author-deposited version published in: http://sam.ensam.eu

Handle ID: .http://hdl.handle.net/10985/9012

\section{To cite this version :}

Stefania CHERUBINI, Jean-christophe ROBINET, Pietro DE PALMA - Nonlinear control of unsteady finite-amplitude perturbations in the Blasius boundary-layer flow - Journal of Fluid Mechanics - Vol. 737, p.440-465 - 2013 


\title{
Nonlinear control of unsteady finite-amplitude perturbations in the Blasius boundary-layer flow
}

\author{
S. CHER U B I N I I , J. - C. R O B I N E T , $^{1}$ \\ P. DE P A L M A ${ }^{2}$ \\ ${ }^{1}$ DynFluid Laboratory, Arts et Metiers ParisTech, 151, Bd. de l'Hopital, 75013 Paris, France \\ ${ }^{2}$ DMMM, CEMeC, Politecnico di Bari, Via Re David 200, 70125 Bari, Italy
}

(Received ?? and in revised form ??)

The present work provides an optimal control strategy, based on the nonlinear NavierStokes equations, aiming at hampering the rapid growth of unsteady finite-amplitude perturbations in a Blasius boundary-layer flow. A variational procedure is used to find the blowing and suction control law at the wall providing the maximum damping of the energy of a given perturbation at a given target time, with the final aim of leading the flow back to the laminar state. Two optimally-growing finite-amplitude initial perturbations capable of leading very rapidly to transition have been used to initialize the flow. The nonlinear control procedure has been found able to drive such perturbations back to the laminar state, provided that the target time of the minimisation and the region in which the blowing and suction is applied have been suitably chosen. On the other hand, an equivalent control procedure based on the linearized Navier-Stokes equations has been found much less effective, being not able to lead the flow to the laminar state when finite-amplitude disturbances are considered. Regions of strong sensitivity to blowing and suction have been also identified for the given initial perturbations: when the control is actuated in such regions, laminarization is observed also for a shorter extent of the actuation region.

The nonlinear optimal blowing and suction law consists of alternated wall-normal velocity perturbations, which appear to modify the core flow structures by means of two distinct mechanisms: i) a wall-normal velocity-compensation at small times; ii) a rotationcounterbalancing effect al larger times. Similar control laws have been observed for different target times, values of the cost parameter, and streamwise extents of the blowing and suction zone, meaning that these two mechanisms are robust features of the optimal control strategy, provided that the nonlinear effects are taken into account.

\section{Introduction}

Delaying transition to turbulence in flows over solid bodies is a fundamental issue for many aerodynamic and industrial applications, such as airplane wings and gas turbine blades. In fact, it is well known that transition to turbulence in boundary-layer flows induces an increase of the skin-friction, causing the enhancement of the drag force over the whole body surface. Recently, in the transportation field, the need for a reduction of the carbon dioxide emissions has stimulated researchers to find solutions to reduce this friction drag using active or passive control devices. Passive control acts by introducing some modifications of the flow aiming at altering its stability properties (for a review, 
see Choi et al. (2008)). For instance, this can be achieved by placing a passive device in regions of strong sensitivity of the flow, which can be identified by global stability tools (see the structural sensitivity analysis by Giannetti \& Luchini (2007); Marquet et al. $(2008 b))$. Such passive strategies are very well-adapted for flows behaving like oscillators (Huerre \& Rossi (1998)), characterized by an intrinsic self-sustained instability at a given frequency, typically arising through a supercritical bifurcation. Oscillators are usually characterized by strongly sensitive wavemaker regions (Giannetti \& Luchini (2007); Marquet et al. $(2008 b))$ where the instability is originated and sustained even in the absence of environmental disturbances. Thus, placing a passive device in such a wavemaker region can result into a stabilisation of the flow. An example of this passive strategy is the suppression of the vortex shedding behind a cylinder by placing a second cylinder close to the separation point (Strykowski \& Sreenivasan (1990)). Passive strategies have been used also for reducing the growth of Tollmienn-Schlichting (TS) waves in boundary-layer flows: by placing small cylindrical devices at the wall, the growth rate of TS waves can be reduced and transition due to their asymptotical growth can be delayed (see Fransson et al. (2006); Shahinfar et al. (2012)). However, in the presence of large environmental disturbances, the boundary-layer presents a very different dynamics, since it behaves like a noise amplifier (for instance, see Marquet et al. (2008a); Alizard et al. (2009)). Noise amplifiers cannot self-sustain oscillations in the absence of an external disturbance source, but they can strongly amplify a broad range of frequencies and spatial scales. Indeed, in the presence of large environmental disturbances, boundary-layer flows are known to experience transition to turbulence at subcritical Reynolds numbers due to the transient amplification of coherent structures such as streamwise vortices and streaks (see Schmid \& Henningson (2001); Brandt et al. (2004)). In these conditions, active control devices are found to be much more suitable to control the transition process, since they may adapt to the environmental unsteady forcing.

Classical active control devices inject fluid (jets) or momentum (plasma actuators) into the flow, following a control law which can be predetermined or computed in real time by a compensator (see the review by Bagheri \& Henningson (2011)). Most of the recent methods for designing compensators aiming at delaying or hampering transition in a boundary-layer flow are based on linear models (for a discussion on linearization, see the review by Kim \& Bewley (2007)). In particular, most of them use the Linear Quadratic Gaussian (LQG) control framework (Lewis \& Syrmos (1995)), which computes a control law based on a linear model of the system and on flow-variable measurements by suitable sensors in the presence of Gaussian noise. The first attempts to use this method to stabilize shear flows has been performed in a simplified framework using Fourier transforms (Bewley \& Liu (1998) for channel flows and Chevalier et al. (2007) for the boundary layer flow). For more complex flows, Fourier modes cannot be used, but the degrees of freedom of the problem need to be reduced as well. This has been accomplished by means of system identification techniques (see the ARMAX model used by Herve et al. (2012) for controlling the flow over a backward-facing step) and projection techniques, such as Galerkin projection onto POD or balanced POD modes (for instance see the work by Barbagallo et al. (2012) for controlling unsteadiness over a rounded backward-facing step, and Semeraro et al. (2011) for a three-dimensional boundary-layer flow).

The applicability of such linear strategies to the control of transition to turbulence is based on the hypothesis that linear models accurately represent the input-output dynamics of the transitional flow systems. For boundary-layer flows, at subcritical Reynolds number, transition can be triggered by the transient growth of perturbations due to the non-normality of the linearized Navier-Stokes operator (Schmid \& Henningson (2001)). The classical bypass transition scenario relies on the linear growth of streamwise streaks; 
these are the linearly optimally-growing structures which could then experience secondary instability and breakdown (see Schoppa \& Hussain (2002); Brandt et al. (2004)). However, starting from the '90s, several studies (see Nagata (1990); Waleffe (1998); Hof et al. (2004); Wedin \& Kerswell (2004); Eckhardt et al. (2007); Viswanath \& Cvitanovic (2009), for instance) have suggested that the path to transition in shear flows is intimately linked to nonlinearity. The dynamical system theory of transition that has been promoted by these authors, among others, relies on the idea that transition and turbulence can be explained as a walk of the system's state among exact coherent states, which are unstable fixed points, periodic orbits or chaotic solutions of the Navier-Stokes equations. Some of these solutions, called edge states, live in the phase-space on the boundary between the laminar and the turbulent attractors, acting as relative attractors for the states evolving along its stable manifold (Skufca et al. (2006); Schneider et al. (2007); Cherubini et al. (2011a); Duguet et al. (2012)). The perturbations living on the laminar-turbulent boundary are very interesting since they can be the most dangerous, being the closest ones to the laminar state capable to trigger transition. Very recently, the problem of finding the minimal energy perturbation on the edge of turbulence has been investigated by solving the nonlinear optimal growth problem for finite-amplitude initial perturbations. This quest has brought to the discovery of nonlinear optimal perturbations, which are characterized by a very different structure with respect to the linear optimal ones and largely outgrow them in energy due to nonlinear mechanisms. The nonlinear optimal perturbation of minimal energy capable of bringing the flow to transition (i.e., the minimal seed of turbulent transition), has been recently found for a pipe flow (Pringle \& Kerswell (2010); Pringle et al. (2012)); a boundary layer flow (Cherubini et al. (2010a, 2011b)); and a Couette flow (Monokrousos et al. (2011); Rabin et al. (2012); Cherubini \& De Palma (2012)). For the boundary-layer and the Couette flow, the minimal seed is characterized by a fundamental invariant structure, composed of a localized array of vortices and low-momentum regions of typical length scale, capable of maximizing the energy growth most rapidly. Cherubini et al. (2011b) has discussed the influence of nonlinear effects in such a strong energy growth, showing that nonlinearity is crucial to sustain the growth of such optimal perturbations. This means that, for mimicking the input-output dynamics of such optimally-growing perturbations, and eventually controlling them, linear models as the ones used in the recent literature (Kim \& Bewley 2007) may be not well-suited. Instead, a nonlinear control strategy should be used.

Very few nonlinear strategies for controlling transition in shear flows have been proposed in the past, mostly due to the complexity of finding a nonlinear reduced-order model able to account for the whole system dynamics, relying only on a few degrees of freedom. To overcome this difficulty, some authors have used a full-state approach based on the fully nonlinear Navier-Stokes equations. For instance, Bewley et al. (2001) have used a direct-adjoint approach minimizing different objective functions aiming at inducing relaminarization of a turbulent channel flow over transpirant walls. Some years later, Chevalier et al. (2002) and Zuccher et al. (2004) have used a similar approach for delaying transition in a boundary-layer flow. However, considering weak initial perturbations and neglecting the influence of the streamwise variation of the base flow, such studies revealed no significant differences with respect to a linear control performed in an equivalent setting. Very recently, a similar approach has been used by Passaggia \& Ehrenstein (2013) to control the two-dimensional instability arising in the flow past a bump, in nonlinearly saturated conditions. In this work, we aim at extending such a nonlinear optimal control approach to the case of a three-dimensional boundary-layer flow in the presence of optimally-growing, finite-amplitude perturbations. An optimal pertur- 
bation is superposed on the Blasius flow; in the absence of a control strategy, due to the very rapid energy growth of such a perturbation, the flow would reach the turbulent state. Our main task is to identify the physical mechanisms which are able to control the energy growth of the optimal perturbation, leading the flow to the laminar state. The control law is computed offline, through an open-loop direct-adjoint approach (Bewley et al. 2001), for each of the considered initial perturbations. In particular, the full-state information about the state is known at $t=0$, so that the control law is optimized for that particular initial state. It is worth to notice that in a practical setting, noise or uncertainties about the state of the system would affect the effectiveness of the control law, which is computed offline. However, the proposed optimal control procedure allows one to determine the best performances achievable by blowing and suction actuators on finite-amplitude unsteady perturbations, and also investigate how the optimal control law would correlate with the coherent structures in boundary-layer transition. Indeed, investigating the damping mechanisms in the optimal case might help to derive practical feedback schemes to employ in realistic configurations (Bewley et al. 2001). With this aim, we compute and analyze the optimal control blowing and suction law for optimally growing initial perturbations; we assess the influence of the nonlinear terms on the effectiveness of the control procedure; and we study the effect of the localisation of the blowing and suction disturbance at wall.

The paper is organized as follows. In the second section we define the problem and describe the minimisation method. In the third section, a thorough discussion of the results of the nonlinear control is provided. In particular, in the first part, the focus is on the effectiveness of the nonlinear control procedure with respect to a linear one, whereas the second part deals with the physical mechanisms inducing laminarization of the flow. Finally, concluding remarks are provided.

\section{Problem formulation}

\subsection{Governing equations and numerical setup}

The behaviour of a three-dimensional incompressible boundary-layer flow is governed by the Navier-Stokes (NS) equations:

$$
\begin{aligned}
& \mathbf{u}_{t}+(\mathbf{u} \cdot \nabla) \mathbf{u}=-\nabla p+\frac{1}{R e} \nabla^{2} \mathbf{u} \\
& \nabla \cdot \mathbf{u}=0
\end{aligned}
$$

where $\mathbf{u}$ is the velocity vector and $p$ is the pressure term (including the contribution of conservative-force fields). Dimensionless variables are defined with respect to the inflow boundary-layer displacement thickness, $\delta^{*}$, and the freestream velocity, $U_{\infty}$, so that the Reynolds number is $R e=U_{\infty} \delta^{*} / \nu, \nu$ being the kinematic viscosity. A computational domain having dimensions equal to $L_{x}=200, L_{y}=20$ and $L_{z}=10.5, x, y$ and $z$ being the streamwise, wall-normal and spanwise directions, respectively, has been employed. The Blasius base flow is obtained by integrating the NS equations with the following boundary conditions: at inlet points, placed at $x_{i n}=200$ downstream of the leading edge of the flat wall, a Blasius boundary-layer profile is imposed for the streamwise and wall-normal components of the velocity vector whereas the spanwise component is set to zero. At outlet points, placed at $x_{\text {out }}=400$ for the reference domain, a standard convective condition is employed (Bottaro (1990)). At the bottom wall, the no-slip boundary condition is prescribed. At the upper-boundary points, the Blasius solution is imposed for the wall-normal component of the velocity, whereas the spanwise velocity component 
and the spanwise vorticity are set to zero. Finally, in the spanwise direction periodicity is imposed for the three velocity components. The NS equations are discretized by a finitedifference fractional-step method using a staggered grid (Verzicco \& Orlandi (1996)). A second-order-accurate centered space discretization is used. After a grid-convergence analysis, a mesh made up by $901 \times 150 \times 61$ points - clustered towards the wall so that the thickness of the first cell close to the wall is equal to 0.1 - is selected for the reference domain. Concerning the resolution at the wall, in the presence of blowing and suction, we have verified that doubling the grid points in the wall-normal direction induces a decrease of the $3 \%$ on the final energy gain value, but the spatial distribution and the temporal variation of the control law $v_{w}(t)$ are essentially unaffected.

\subsection{Nonlinear control}

The nonlinear behaviour of a perturbation $\mathbf{q}=\left(u^{\prime}, v^{\prime}, w^{\prime}, p^{\prime}\right)^{T}$ evolving in a laminar incompressible flow over a flat plate is studied by employing the NS equations written in a perturbative formulation, with respect to the two-dimensional Blasius steady state solution, $\mathbf{Q}=(U, V, 0, P)^{T}$. A zero perturbation condition is chosen for the three velocity components at the $x$-constant and $y$-constant boundaries, except for the blowing and suction slot, where a Dirichlet condition is imposed for the wall-normal velocity. Periodicity of the perturbation is imposed in the spanwise direction. The zero perturbation condition at inflow and outflow points is enforced by means of a fringe region (Cherubini et $a l .(2010 b)$ ), which allows the perturbation at the exit boundary to vanish smoothly.

The aim of the procedure is to prevent the transition to turbulence of a given perturbation, using a full-state control strategy based on a direct-adjoint formulation. In order to make the control realistic, the actuators are placed at the wall along a streamwiselocalized disturbance slot $S_{w}$, placed on the flat plate, as shown in Figure 1. The blowing and suction law is provided by prescribing the wall-normal velocity at the disturbance slot, $v_{w}$, which varies in time according to the results of the minimization detailed below. Different configurations of the blowing and suction slot have been considered. Firstly, a single disturbance strip has been placed at seven different streamwise positions in order to study the sensitivity of the blowing and suction law with respect to the initial perturbation: for case 0, $215<x<280$; for case I, $215<x<247.5$; for case II, $247.5<x<280$; for case III, $215<x<231.25$; for case IV, $231.5<x<247.5$; for case V, 247.5 $<x<263.75$; for case VI, $263.75<x<280$. Then, five equally spaced disturbance strips have been considered in the zone $215<x<280$, each one having length $l_{x}=1.5$ (case VII, see the bottom frame of figure 1 ). The different localisation of the strips allows one to take into account the convective character of the perturbations we aim to control, as well as to verify in which region, and/or in which phase of their growth the considered perturbations are most sensitive to suction and blowing at the wall.

The minimization aims at finding the blowing and suction law of lowest cost providing the lowest disturbance energy at a given target time, $T$. The disturbance energy is defined as

$$
E(t)=\int_{V}\left[\mathbf{u}^{\prime}(t) \cdot \mathbf{u}^{\prime}(t)\right] d V,
$$

where $V$ is the volume of the whole computational domain. A Lagrange multiplier technique is used (Zuccher et al. 2004; Pringle \& Kerswell 2010), aiming at finding the blowing and suction law at the wall, $v_{w}(t)$, satisfying the following two conditions:

(a) the integral energy over the time interval $(0, T)$ must be minimum, the blowing 


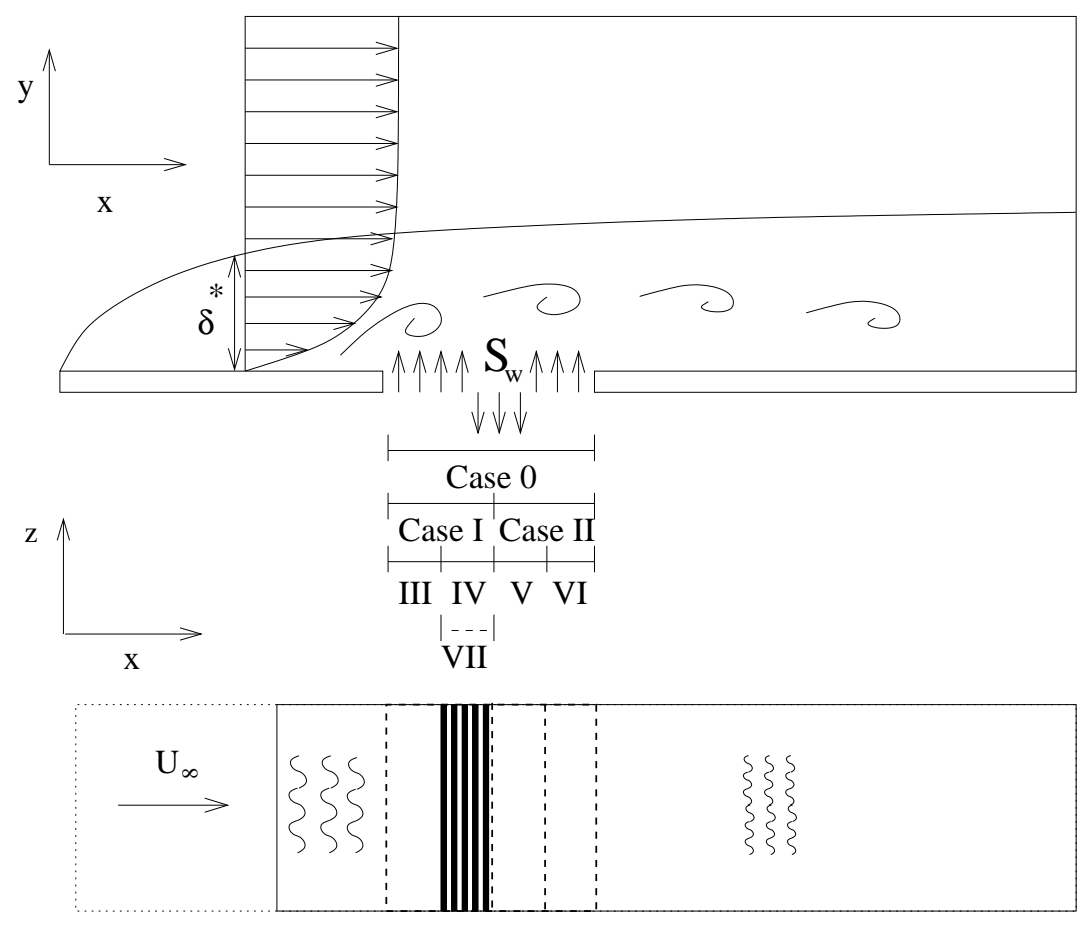

FIGURE 1. Flow configuration and layout of the control slot.

and suction energy on the disturbance slot being:

$$
E_{w}(t)=\int_{S_{w}}\left[v_{w}(t)\right]^{2} d S_{w}
$$

(b) at target time $T$, the energy gain of the perturbations, $E(T) / E(0)$, must be minimum.

Thus, the objective function of the minimization procedure, $\Im$, is the energy gain of the perturbations plus the cost of the control (see also Passaggia \& Ehrenstein (2013)):

$$
\Im=\frac{E(T)}{E(0)}+\gamma^{2} \int_{0}^{T} E_{w}(t) d t
$$

where $\gamma^{2}$ is a parameter setting the weight of the control cost with respect to the energy gain. The Lagrange multiplier technique consists in searching for extrema of an augmented functional, $\mathcal{L}$, with respect to every independent variable. The augmented functional is given by the objective function (2.4) plus the following constraints:

(a) the perturbation $\mathbf{q}^{\prime}$ satisfies the three-dimensional incompressible NS equations, written in a perturbative formulation;

(b) the shape and amplitude of the initial perturbation superposed to the base flow is given by the initial velocity vector $\mathbf{u}_{0}^{\prime}=\left(u_{0}^{\prime}, v_{0}^{\prime}, w_{0}^{\prime}\right)^{T}$;

(c) the wall-normal velocity at the disturbance slot equals the blowing and suction law found by the minimization $\left(v^{\prime}=v_{w}\right.$ on the disturbance slot).

(d) the blowing and suction has zero net mass flux at every instant of time, so that the integral of $v_{w}$ at the disturbance slot surface is zero. 
Thus, the functional is written as:

$$
\begin{aligned}
\mathcal{L} & =\Im-\int_{0}^{T} \int_{V} a\left(u_{x}^{\prime}+v_{y}^{\prime}+w_{z}^{\prime}\right) d V d t \\
& -\int_{0}^{T} \int_{V} b\left[u_{t}^{\prime}+v^{\prime} U_{y}+V u_{y}^{\prime}+\left(u^{\prime} U+u^{\prime} u^{\prime}\right)_{x}+\left(u^{\prime} v^{\prime}\right)_{y}+\left(u^{\prime} w^{\prime}\right)_{z}+p_{x}^{\prime}-\frac{\left.u_{x x}^{\prime}+u_{y y}^{\prime}+u_{z z}^{\prime}\right]}{R e}\right] d V d t \\
& -\int_{0}^{T} \int_{V} c\left[v_{t}^{\prime}+U v_{x}^{\prime}+u^{\prime} V_{x}+\left(u^{\prime} v^{\prime}\right)_{x}+\left(v^{\prime} v^{\prime}+v^{\prime} V\right)_{y}+\left(v^{\prime} w^{\prime}\right)_{z}+p_{y}^{\prime}-\frac{v_{x x}^{\prime}+v_{y y}^{\prime}+v_{z z}^{\prime}}{R e}\right] d V d t \\
& -\int_{0}^{T} \int_{V} d\left[w_{t}^{\prime}+U w_{x}^{\prime}+V w_{y}^{\prime}+\left(w^{\prime} u^{\prime}\right)_{x}+\left(w^{\prime} v^{\prime}\right)_{y}+\left(w^{\prime} w^{\prime}\right)_{z}+p_{z}^{\prime}-\frac{\left.w_{x x}^{\prime}+w_{y y}^{\prime}+w_{z z}^{\prime}\right]}{R e}\right] d V d t \\
& -\Phi \int_{0}^{T} \int_{S_{w}}\left(v^{\prime}-v_{w}\right) d t d S_{w}-\Lambda \int_{0}^{T}\left[\int_{S_{w}} v_{w}(t) d S_{w}-0\right] d t \\
- & \lambda_{u}\left[\left(u_{0}^{\prime}-u^{\prime}(0)\right)\right]-\lambda_{v}\left[\left(v_{0}^{\prime}-v^{\prime}(0)\right)\right]-\lambda_{w}\left[\left(w_{0}^{\prime}-w^{\prime}(0)\right)\right]
\end{aligned}
$$

where $a, b, c, d, \Phi, \Lambda, \lambda_{u}, \lambda_{v}, \lambda_{w}$ are Lagrange multipliers. Integrating by parts and setting to zero the first variation of $\mathcal{L}$ with respect to $u^{\prime}, v^{\prime}, w^{\prime}, p^{\prime}$ leads to the adjoint equations:

$$
\begin{aligned}
-b_{t} & =2 b_{x} u^{\prime}+b_{y} v^{\prime}+b_{z} w^{\prime}+b_{x} U+(b V)_{y}-c V_{x}+c_{x} v+d_{x} w+a_{x}+\frac{b_{x x}}{R e}+\frac{b_{y y}}{R e}+\frac{b_{z z}}{R e} \\
-c_{t} & =c_{x} u^{\prime}+2 c_{y} v^{\prime}+c_{z} w^{\prime}+(c U)_{x}+c_{y} V-b U_{y}+b_{y} u+d_{y} w+a_{y}+\frac{c_{x x}}{R e}+\frac{c_{y y}}{R e}+\frac{c_{z z}}{R e} \\
-d_{t} & =d_{x} u^{\prime}+d_{y} v^{\prime}+2 d_{z} w^{\prime}+d U_{x}+(d V)_{y}+b_{z} u+c_{z} v+a_{z}+\frac{d_{x x}}{R e}+\frac{d_{y y}}{R e}+\frac{d_{z z}}{R e} \\
0 & =b_{x}+c_{y}+d_{z}
\end{aligned}
$$

where $\mathbf{q}^{\dagger}=(a, b, c, d)^{T}$ is defined as the adjoint vector. The adjoint equations are linked to the direct ones by the presence of direct variables in the advection terms. By using the boundary conditions of the direct problem and by taking:

$$
\begin{aligned}
& b=0, c=0, d=0, \text { for } \quad y=0 \quad \text { and } \quad y=L_{y}, \\
& b=0, c=0, d=0, \text { for } \quad x=x_{\text {in }} \quad \text { and } \quad x=x_{\text {out }},
\end{aligned}
$$

an expression for the Lagrange multiplier $\Phi$ is found:

$$
\Phi=-\Lambda-a-\frac{c_{y}}{R e}
$$

which is used to determine an equation for the gradient of the functional with respect to $v_{w}$. Moreover, the compatibility conditions (Zuccher et al. 2004) are:

$$
\frac{2 u^{\prime}}{E_{0}}-b=0, \frac{2 v^{\prime}}{E_{0}}-c=0, \frac{2 w^{\prime}}{E_{0}}-d=0, \text { for } t=T .
$$

The direct and adjoint equations are parabolic in the forward and backward time direction, respectively, so that they can be solved by a coupled iterative approach, similar to the one used by Cherubini et al. (2010b). By solving the direct and adjoint equations at each step of the iterative procedure, the first variation of the augmented functional with respect to $\mathbf{q}$ and $\mathbf{q}^{\dagger}$ is set to zero. Moreover, the gradient of $\mathcal{L}$ with respect to the blowing and suction variable $v_{w}$ has to vanish within a reasonable number of iterations. In order to achieve convergence efficiently, a steepest descent algorithm is used. The initial state is updated in the steepest descent direction, namely the direction opposed to the gradient:

$$
\frac{\partial \mathcal{L}}{\partial v_{w}}=2 \gamma^{2} v_{w}-\Phi=2 \gamma^{2} v_{w}+a+\Lambda+\frac{c_{y}}{R e},
$$


with an adjustable step length $\alpha$, so that $v_{w}^{(n+1)}=v_{w}^{n}-\alpha^{n} \frac{\partial \mathcal{L}^{n}}{\partial v_{w}}$. The step length $\alpha$ has been chosen carefully in order to ensure convergence to the optimal value, as described below, with values in the range $[0.01-0.1]$.

The optimization procedure for a chosen target time $T$ can be summarized as follows:

(a) An initial guess is made for the control law, $v_{w}(t)$, and a given initial perturbation is superposed on the base flow.

(b) The direct problem (the NS equations) is integrated up to $t=T$.

(c) The adjoint variables, $b(T), c(T), d(T)$, are provided by the compatibility conditions (2.9).

(d) The adjoint problem (2.6) is integrated backward in time from $t=T$ to $t=0$.

(e) At $t=0$, the control law is updated in the steepest descent direction with step length $\alpha$.

(f) Convergence is assessed:

(i) if the decrease of the objective function between two successive iterations and the value of the gradient are smaller than a chosen threshold, the loop is stopped, otherwise the procedure is continued from step $(b)$;

(ii) if an increase of the objective function is found, the value of $\alpha$ is halved.

Each iteration requires the integration of the three-dimensional Navier-Stokes and adjoint equations forward and backward in time up to the target time; moreover, due to the presence of the direct variables in the advection terms of the adjoint equations, the whole direct-variable field needs to be stored at each time step, requiring a remarkable storage capacity. Considering that about 100 iterations are needed to achieve convergence within machine accuracy, the computational effort required to optimize the solution for a single set of the independent parameters $\left(T, R e, \gamma^{2}, \mathbf{u}_{0}\right.$, and the size of the disturbance slot) is very high. To keep the computational cost affordable, the optimizations have been performed up to a target time $T=125$. The convergence is very smooth, as one can see in Figure 2 which shows plots of the residual $e=\left(\Im^{n+1}-\varsigma^{n}\right) / \varsigma^{n}$, the gradient of the functional and the energy gain $E(T) / E(0)$. One can see that the gradient decreases smoothly up to machine zero in about 100 iterations. Similar convergence curves are obtained for different parameters and initial perturbations, although convergence have been found easier to achieve in the case of strongly localized initial perturbation (i.e., nonlinear optimal) than in more extended (linear optimal) ones. It is noteworthy that for the considered problem, convergence of the Lagrange multiplier method is much easier to achieve than an equivalent energy gain maximization (see Cherubini et al. (2011b)). This is probably due to the fact that here we are targeting a state close to the laminar one, whereas an energy maximization performed with the same parameters targets a state which is close to chaos, and thus much more difficult to converge to.

\section{Results}

\subsection{Performance of the control strategy}

The nonlinear control has been applied to a boundary-layer flow with a supercritical Reynolds number, $R e=610$ (the critical Reynolds number for the Blasius boundary layer being $R e \approx 520$ ). Since the boundary-layer flow is characterized by convective instabilities, we are seeking to control impulsive perturbations superposed at the initial time, $t=0$, on the Blasius base flow. Two types of initial perturbations have been considered, namely, a linear and a nonlinear optimal perturbation, defined as the perturbations of the base flow which induce the largest energy growth at a given target time, in a linear and a nonlinear framework, respectively. These perturbations have been computed by 


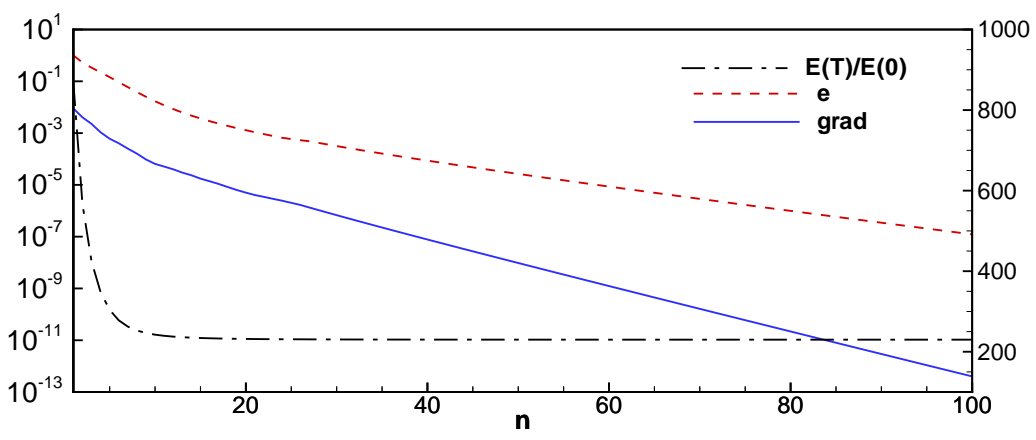

Figure 2. Gradient of the functional (solid line), residual (dashed line) and energy gain (dashed-dotted line) versus the number of iterations for case 0 with $T=100, \gamma^{2}=0.2$.

means of an optimization of the perturbation energy gain using the linearized and the nonlinear Navier-Stokes equations, respectively (see Cherubini et al. $(2010 b, 2011 b)$ ). In both cases, the initial energy of the optimal perturbations is chosen in order to achieve transition. Since in Cherubini et al. (2011b) it has been observed that linear optimal perturbations need larger initial energies to achieve transition than nonlinear optimal ones, a linear optimal perturbation (LOP) with $E(0)=0.1$ and a nonlinear optimal perturbation (NLOP) having an initial energy equal to $E(0)=0.01$ have been selected. The two perturbations are shown in Figure 3, which provides the streamwise vorticity and velocity isosurfaces. Both perturbations show alternated quasi-streamwise vortices and patches of streamwise velocity perturbations, but the following differences are observed: i) the nonlinear perturbation is much more localized in space; ii) it is characterized by a large amplitude of the streamwise velocity perturbation; iii) its vortices are streamwiseinclined. Both perturbations evolve in time initially amplifying themselves via the Orr and the lift-up mechanisms. In particular, the LOP induces an array of streaks alternated in the streamwise and spanwise directions, shown in the left frame of figure 4, which then experience secondary instability of both varicose and sinuous type (see Cherubini et al. $(2010 b))$. On the other hand, the evolution of the NLOP is more complex, since the dislocations of the initial localized patches of finite amplitude streamwise disturbance generate $\Lambda$-shaped structures of slow and fast fluid (see the green surfaces on the right frame of figure 4), while the vortices are further inclined, sustained by nonlinear coupling, and stretched by the mean flow, creating $\Lambda$ and hairpin vortices of finite amplitude (see Cherubini et al. (2011b) for further details). Such vortical structures release smaller scale vortices and hairpin structures (see also Adrian (2007)) leading to turbulence in a very fast way.

The initial optimal perturbations described above have been superposed on the base flow at $t=0$, and the control procedure has been used to compute the optimal blowing and suction law achieving the maximum reduction of the perturbation energy at a given target time. Figures 5 (a) and (b) show the value of the energy gain versus the target time obtained for both perturbations in the uncontrolled (dashed lines) and the nonlinearly controlled case, with $\gamma^{2}=0.2$, using the largest blowing and suction slot (case 0 ). In the uncontrolled case (dashed lines), one can observe that the NLOP (figure 5 (a)) induces a very large energy gain in a very short time, whereas the LOP (figure 5 (b)) leads to a plateau of the energy value corresponding to the phase of streak saturation, before the streaks experience secondary instability. In the controlled case (solid lines), for both initial perturbations a large decrease of the energy gain is observed for large enough target 

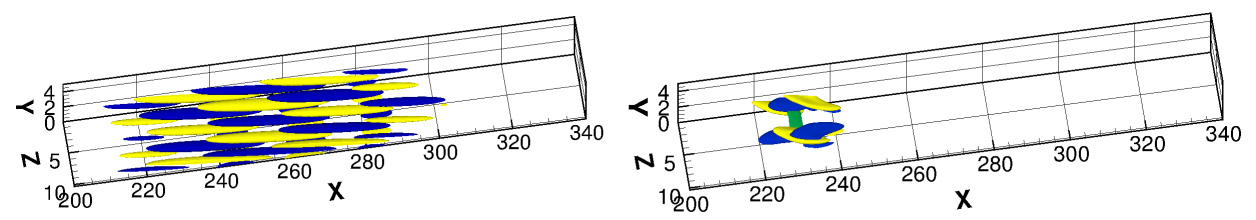

FIGURE 3. Isosurfaces of the streamwise velocity (green) and vorticity (yellow and blue) for a linear (left) and a nonlinear (right) optimal perturbation at $t=0$ computed for $T=75$, with initial energies $E_{0}=0.01$ and $E_{0}=0.1$, respectively.
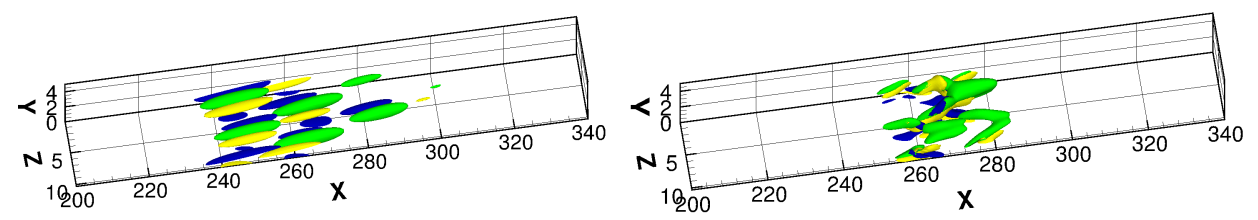

FIGURE 4. Isosurfaces of the streamwise velocity (green) and vorticity (yellow and blue) for a linear (left) and a nonlinear (right) optimal perturbation at $t=T=75$ computed with the initial energies $E_{0}=0.01$ and $E_{0}=0.1$, respectively.

times, i.e., for $T>50$, meaning that the control has to operate on a time scale larger than 50 units to induce a significant effect on the perturbation evolution (a similar behaviour has been found by Bewley et al. (2001) for a turbulent channel flow). In particular, for the NLOP (Figure 5 (a)) the control becomes very effective at large target times, inducing a decrease of one order of magnitude of the energy gain for the largest target time, $T=125$. On the other hand, for the LOP, a maximum decrease of the energy gain of a factor 3 is observed.

For both initial perturbations, it is interesting to evaluate the effect of the nonlinear terms in the procedure for determining the control law. Thus, for comparison, we have performed a linearized direct-adjoint control for both initial perturbations, using the same parameters employed in the nonlinear procedure but imposing the linearized NavierStokes equations as constraint. The evolution of the energy gain versus the target time is provided in Figure 6, for the NLOP (a) and the LOP (b), in the linear controlled (solid lines) and uncontrolled (dashed lines) cases. One can notice that in both cases the linear control is less effective than the fully nonlinear control, achieving a maximum reduction of the energy gain of $30 \%$ for an initial LOP, and less than $8 \%$ for the NLOP. It is also noteworthy that in the linearized case the energy gain achieved by the NLOP is much lower than in the fully nonlinear case (compare Figures 5 and 6), whereas the linear optimal one reaches energy values similar to the previous ones for $T<100$, and then it starts to decay. This could be anticipated since it is known that streaks grow due to a linear mechanisms but nonlinear effects are crucial to sustain the coherent structures and to complete the transition process (Waleffe 1997).

We have thus shown that the nonlinear control law is much more effective than a linear one to reduce the energy of finite-amplitude perturbations. In order to verify if such a reduction corresponds indeed to a laminarization of the flow, we have performed several DNSs in the controlled and uncontrolled cases and analyzed the time evolution of the energy gain and of the maximum value of the streamwise velocity component. The uncontrolled and controlled cases corresponding to three different target times, $T=75$, $T=100$, and $T=125$, are shown in Figure 7 for the NLOP (the cases with $T<75$ are not shown since they only slightly differ from the uncontrolled case). The energy 


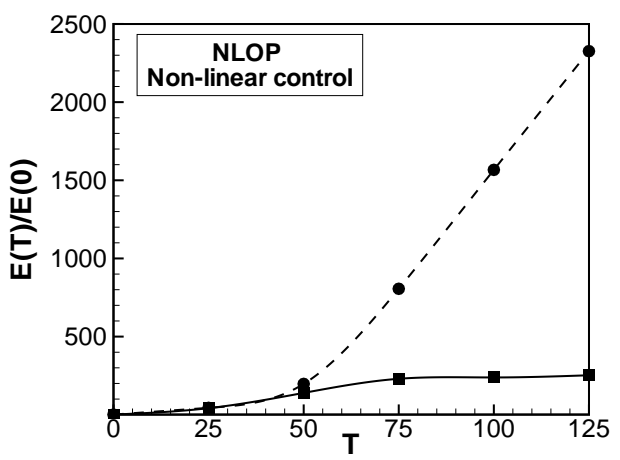

(a)

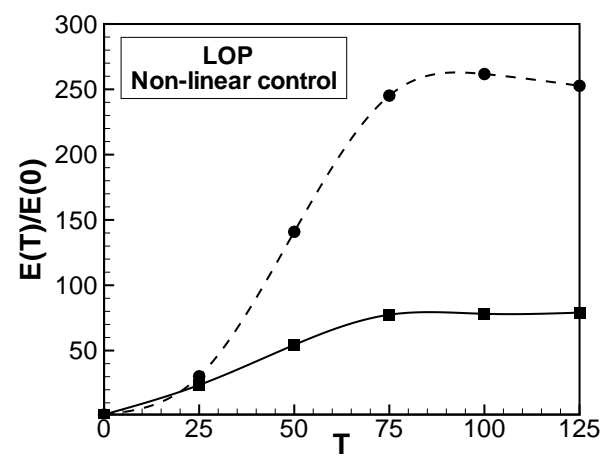

(b)

FIGURE 5. Energy gain versus target time $T$ for (a) the nonlinear optimal perturbation and (b) the linear optimal one, in the uncontrolled (dashed lines) and in the nonlinearly controlled case 0 with $\gamma^{2}=0.2$ (solid lines).

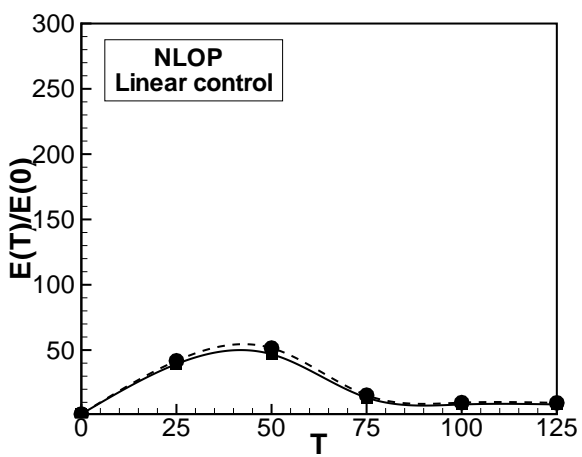

(a)

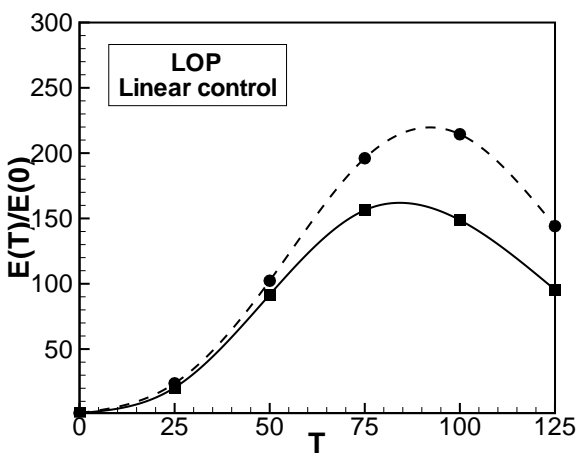

(b)

FiguRE 6. Energy gain versus target time $T$ obtained by a linearized minimisation for an initial (a) nonlinear and (b) linear optimal perturbation: linear uncontrolled (dashed lines) and linearly controlled case 0 with $\gamma^{2}=0.2$ (solid lines).

gain (Figure 7 (a)) appears to slowly grow up to $t \approx 200$, and then begins to decrease (notice that the perturbation begins to be convected out of the computational domain for $t>300)$. On the other hand, the maximum value of the streamwise velocity component (Figure $7(\mathrm{~b}))$ begins to decrease at shorter times $(t \approx 100)$. In fact, even if the maximum perturbation amplitude begins to decrease at $t \approx 100$, its spreading in the streamwise and spanwise directions induces a slight increase of the energy up to $t \approx 200$ (see the description of the disturbance evolution in the next section).

A similar behaviour has been observed for the LOP, as shown in Figure 8 (a) and (b), for the energy gain and the maximum value of the streamwise disturbance, respectively. In this case, since the LOP needs a larger time interval to induce transition, in order to avoid the perturbation to be convected out of the computational domain before transitioning to turbulence, the domain length has been doubled in the streamwise direction. One can observe that also in this case the nonlinear control leads to laminarization effectively. One can also notice that for the LOP, the time needed by the control law to induce a reduction 


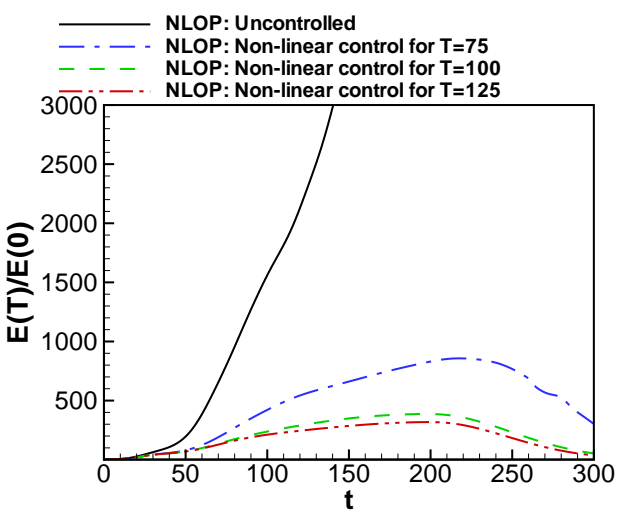

(a)

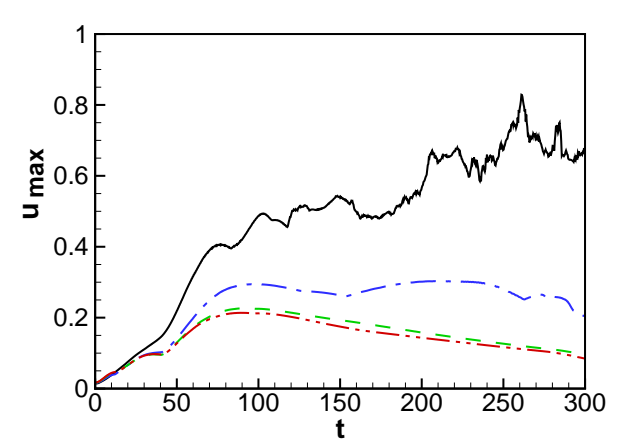

(b)

Figure 7. Time evolution of the energy gain (a) and of the maximum value of the streamwise perturbation velocity (b) extracted by a DNS initialized by the NLOP in the uncontrolled (solid line) and in the nonlinearly controlled case 0 with $\gamma^{2}=0.2 ; T=75$ (dashed-dotted), $T=100$ (dashed), and $T=125$ (dashed-dotted-dotted).

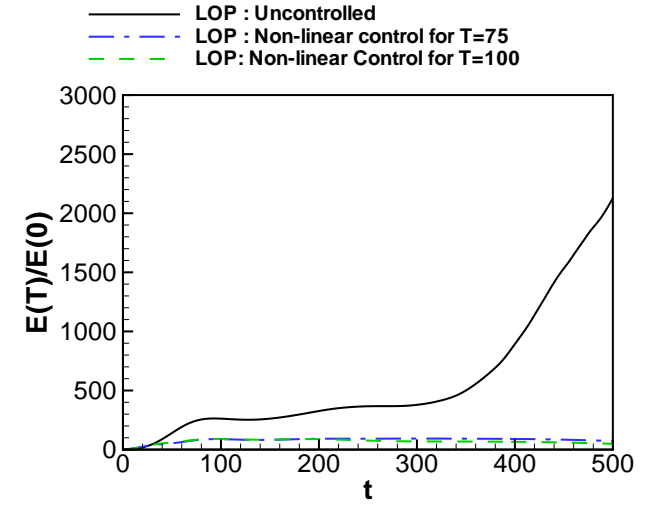

(a)

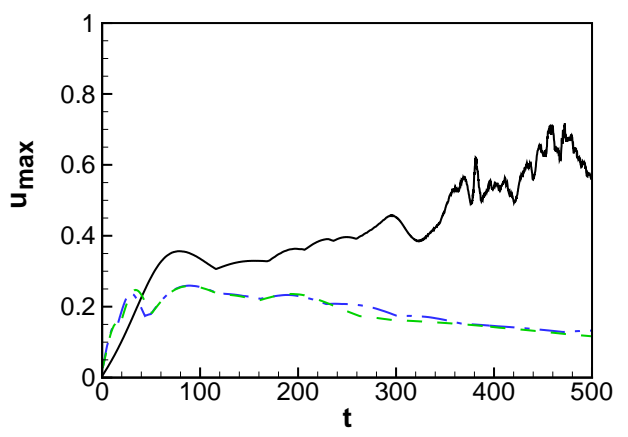

(b)

Figure 8. Time evolution of the energy gain (a) and of the maximum value of the streamwise perturbation velocity (b) extracted by a DNS initialized by the LOP in the uncontrolled (solid line) and in the nonlinearly controlled case 0 with $\gamma^{2}=0.2 ; T=75$ (dashed-dotted) and $T=100$ (dashed).

of the energy is larger than for the NLOP, probably because the initial disturbance is more extended in space.

The effect of the control law obtained by the linearized direct-adjoint approach on a fully nonlinear direct simulation is shown in Figure 9. One can observe that, for the NLOP (Figure 9 (a)), the effectiveness of the linear control is very poor since it induces but slight modifications on the evolution of the perturbation amplitude. In principle, this could be due either to the spatial distribution of the control law or to its amplitude (which is found to be lower with respect to the nonlinear case). To verify this, we have scaled the amplitude of $v_{w}$ of a factor 10 and then 100, reaching amplitudes similar to the ones found in the nonlinear case. In the first case, a slight decrease of the streamwise velocity component amplitude is observed for $100<t<200$, but transition to turbulence is reached almost at the same time than in the uncontrolled case (solid line). For a rescaling factor of 100, the perturbation strongly increases and reaches transition much 


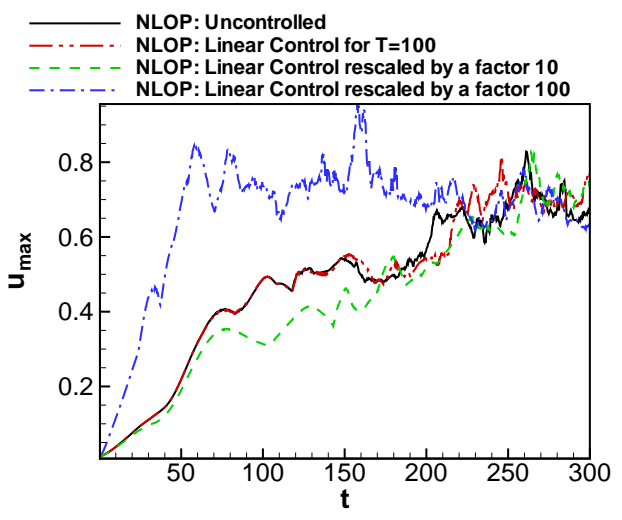

(a)

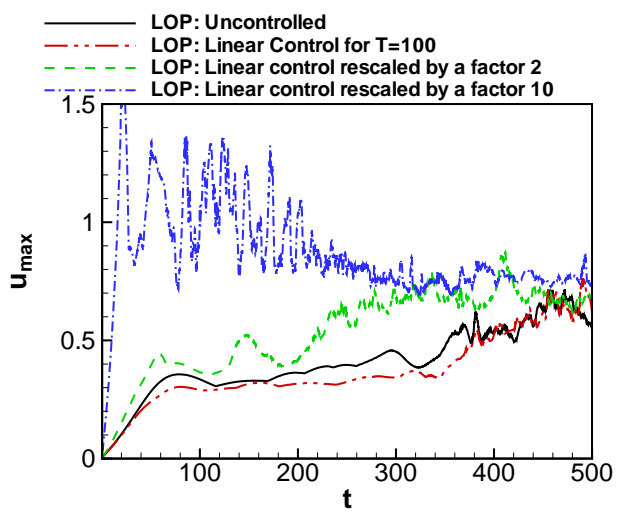

(b)

Figure 9. Time evolution of the maximum value of the streamwise perturbation velocity extracted by a DNS initialized by the (a) NLOP and the (b) LOP, in the uncontrolled (solid line) and in several linearly controlled cases (see the legend), for case 0 with $\gamma^{2}=0.2$ and $T=100$.

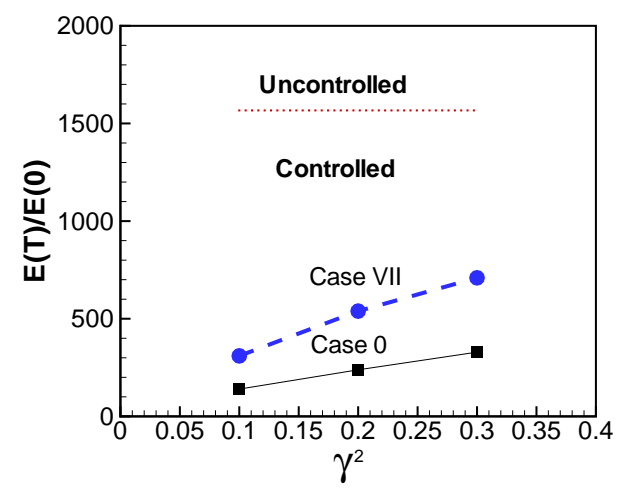

(a)

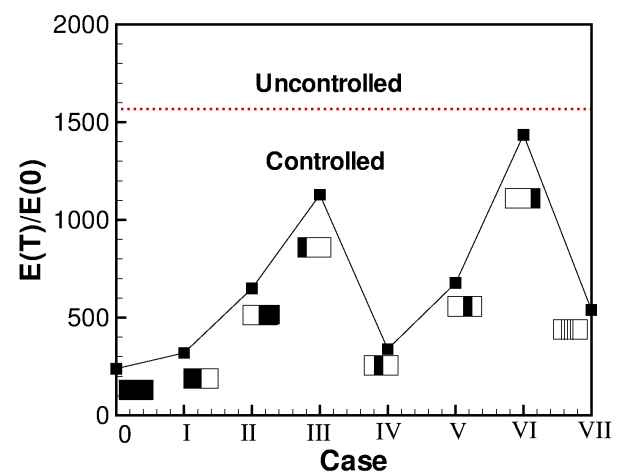

(b)

FIGURE 10. Energy gain for a NLOP in the presence of a nonlinear control law obtained for $T=100$ versus: (a) different values of $\gamma^{2}$; (b) different lengths and positions of the blowing and suction with $\gamma^{2}=0.2$. The black and white rectangles indicate the position of the disturbance slot with respect to case 0 .

earlier (at $t \approx 50$ ). Thus, it appears that, even if the amplitude of the control law has a certain influence on the evolution of the disturbance, the linear control is not effective in inducing laminarization because of its particular shape. Similar observations can be made concerning the LOP, the linear control being partially able to damp the initial growth of the perturbation, but eventually not driving the flow to the laminar state, as shown in figure 9 (b).

Once the effect of the initial disturbance and of the nonlinear terms has been analyzed, it can be interesting to study the influence of the cost parameter $\gamma^{2}$ on the performance of the control. For this analysis, we choose as reference case the nonlinear control at $T=100$, since for $T \geqslant 100$ the control law does not show remarkable variations (see figure 7 ), for case 0 . The black squares in figure 10 (a) show the energy gain achieved in the reference case by a NLOP in presence of a control law obtained for three values of $\gamma^{2}$. Obviously, a larger decrease in energy gain due to the control is obtained for lower values of $\gamma^{2}$ (i.e., for a cheaper actuator). Moreover, the controlled energy shows a linear slope 


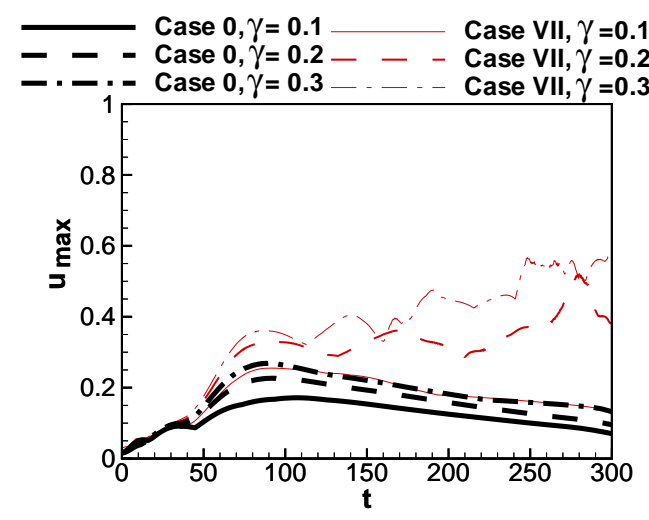

(a)

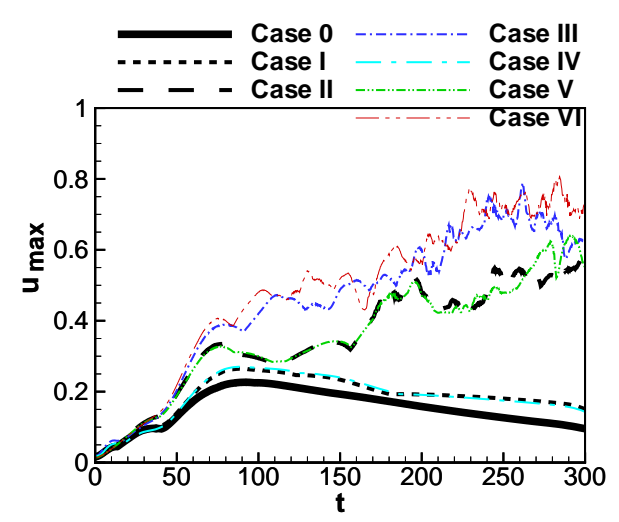

(b)

FIgURE 11. Time evolution of the maximum value of the streamwise perturbation velocity extracted by a DNS initialized by the NLOP in the presence of a nonlinear control law obtained for $T=100$ and (a) three different values of $\gamma^{2}$ for case 0 and case VII; (b) different positions and length of the disturbance strip from case 0 to VI with $\gamma^{2}=0.2$.

with respect to this parameter, indicating that we can estimate the value of $\gamma^{2}$ needed to obtain a given energy gain at a given target time. The circles in figure 10 (a) show that the same linear trend (but with a different slope) is found also for a different localization of the disturbance strips (case VII). Figure 11 (a) shows the maximum value of the streamwise velocity perturbation extracted by six controlled DNSs, performed for case 0 and case VII, for the three values of $\gamma^{2}$ considered here. For case 0 (black thick lines), the active control is able to lead the flow to laminarization for all of the considered values of $\gamma^{2}$. Whereas, in the case of the localized disturbance strips (case VII, red thin lines), laminarization is obtained only for the cheapest control (the lowest value of $\gamma^{2}$ ). Moreover, if the five localized disturbance strips are equally distributed in the disturbance slot, a further reduction of the control cost is needed to achieve laminarization. This indicates that the length and localization of the disturbance strip has an important role for the effectiveness of the control.

Thus, we analyze the influence of the position and shape of the disturbance strip on the performance of the control. Figure 10 (b) shows the energy gain at target time $T=100$ obtained for cases 0 to VII (the black and white rectangles indicating the length of the disturbance strips with respect to case 0 configuration). One can observe that the controlled energy gain changes slightly when the blowing and suction is active in the first half (case I) or in the second quarter (case IV) of the disturbance strip. Whereas, the energy gain strongly increase when the control is applied in the second half (cases II, $\mathrm{V}, \mathrm{VI}$ ) or in the first quarter (case III). This means that the region in which the flow is most sensitive to blowing and suction is the second quarter of the reference disturbance strip $(247.5<x<280)$. Whereas, when the blowing and suction is applied only in the second half or in the first quarter, the control procedure is much less effective. For this reason, for case VII, the five localized blowing and suction strips have been placed in the second quarter of the reference disturbance slot, namely in the most sensitive zone for the NLOP. The final controlled energy gain is about 2 times that found in the reference case, and 1.5 times the one found in case IV (second quarter). However, changing the value of $\gamma^{2}$, and thus increasing the energy of the blowing and suction, we can achieve an energy gain at target time which is close to the one found in the reference case, as provided by the dashed line in Figure 10 (a). Direct numerical simulations confirm these 
findings, showing that the flow laminarizes only when the control is applied in the first half or the first quarter of the disturbance strip, which correspond to the regions in which the control law (for case 0) is characterized by a higher amplitude, as it will be shown in detail in the next subsection. The time evolution of the maximum value of the streamwise velocity component is provided in Figure 11 (b) for cases from 0 to VI, and $\gamma^{2}=0.2$. One can observe that the $u_{\max }$ curves are very close for case I and III, meaning that the disturbance is only slightly affected by the position of the blowing and suction when applied in the first quarter of the disturbance strip. These results will be discussed in the next section, where the analysis of the flow structures with respect to the applied control is provided.

\subsection{Analysis of the flow structures}

In this section we analyze the shape of the blowing and suction law and its effect on the evolution of the perturbation in the flow. We have chosen as reference case the nonlinear control initialized by the NLOP, case 0 , with $T=100$ and $\gamma^{2}=0.2$. Figure 12 provides the contours of $v_{w}$, extracted at $t=5, t=20$ and $t=40$. One can observe localized patches of wall-normal velocity, alternated in the streamwise and spanwise directions, initially showing a quasi circular shape, and eventually turning into $\Lambda$ structures. For larger times $(t>70)$ the actuation fades away. Therefore, the control law is very localized in space and time, and it hampers the growth of the perturbation in its first phases. This explains the high sensitivity of the initial perturbation to blowing and suction in the second quarter of the disturbance slot, which is the region where the NLOP is localized at small times (for $t<20$ ). On the other hand, when the optimal control is determined by the linearized Navier-Stokes equations, a very different blowing and suction law has been found. Figure 13 (a) shows the contours of $v_{w}$ for a linear optimal control computed for the same conditions of the reference case. One can observe that the $v_{w}$ patches lose their localized shape and strongly elongate in the streamwise direction. One can also observe, by comparing figures 13 (a) and (b), that such contours are rather similar to the ones recovered for an initial linear optimal perturbation (both for a linear control), even if the shape of the initial perturbation is very different. This means that considering nonlinear effects for determining a control law is crucial when dealing with finite amplitude perturbations, since a linear model would predict an evolution very different from the real dynamics of the perturbation.

In order to establish a link between the blowing and suction law and the time evolution of the perturbation structure, snapshots of the disturbance field have been analyzed. Figures 14 (a), (b), and (c) show the snapshots at $t=20, t=40$, and $t=60$, respectively, in the controlled reference case. At $t=20$, the rounded-shaped patches of $v_{w}$ (coloured shaded contours) are localized in correspondence of the flow regions with large values of the wall-normal velocity (black and white isosurfaces). In particular, we can observe that a positive (negative) $v_{w}$ at the wall lies in correspondence with a negative (positive) patch of wall-normal velocity perturbation within the flow field. Thus, we can infer that the first effect of the nonlinear control is to try to cancel out the wall-normal finite amplitude perturbation injected within the flow by using an opposite velocity at the wall, similarly to the opposition control technique (see Hammond et al. (1998) for instance). Moreover, one can notice that the blowing and suction at $t=20$ is localized in the second quarter of the disturbance slot, which has been found to be the most sensitive region for the NLOP. Thus, it appears that the mechanism of compensation of the wallnormal velocity by blowing and suction is crucial for the performance of the control. Probably this is due to the low amplitudes of the perturbations at small times which allow the control to be effective at a small cost (for instance, the maximum value of $v$ 

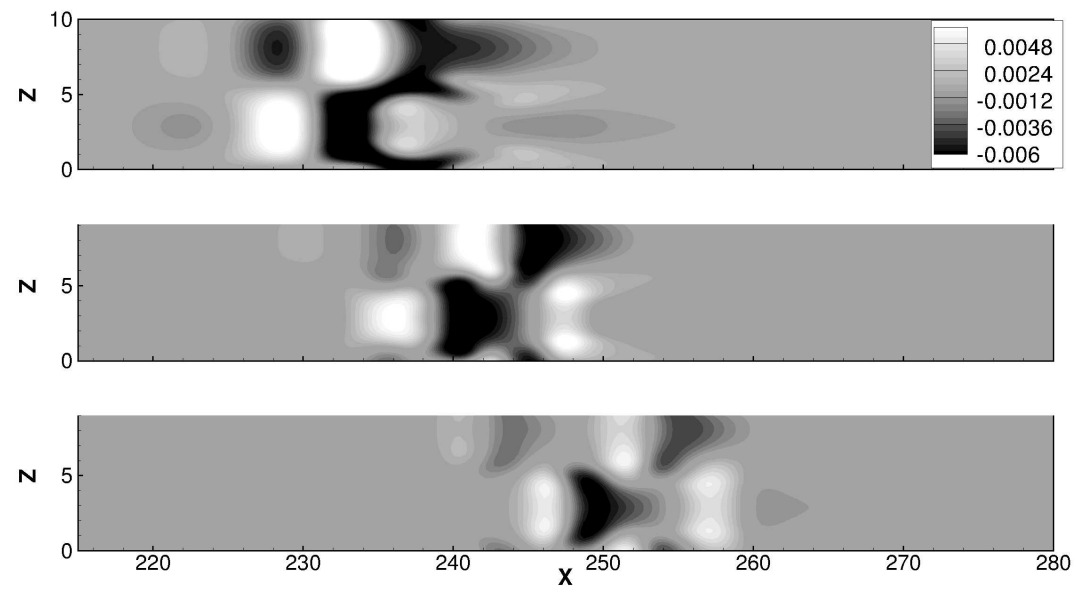

Figure 12. Snapshots of the blowing and suction velocity $v_{w}$ at the wall at $t=5$, (top frame) $t=20$ (middle frame), $t=40$ (bottom frame) for the reference case.

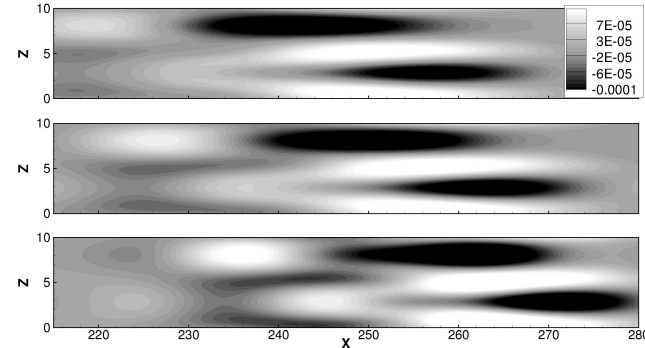

(a)

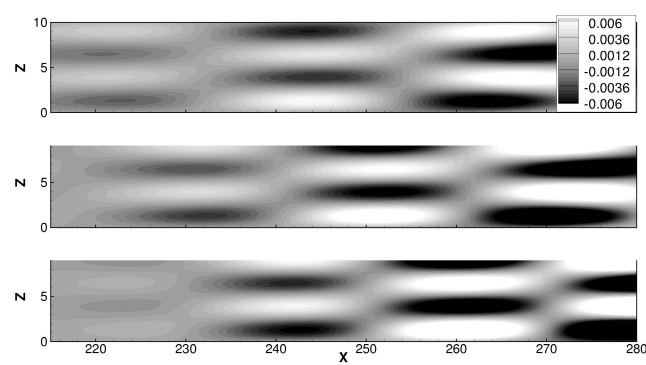

(b)

Figure 13. Snapshots of the blowing and suction velocity $v_{w}$ at the wall at $t=5$, (top frames) $t=20$ (middle frames), $t=40$ (bottom frames) for a linear control, case 0 , with $T=100$, $\gamma^{2}=0.2$ initialized by the nonlinear (a) and the linear (b) optimal perturbations.

at $t=20$ is $v_{\max } \approx 0.023$ in the uncontrolled case, whereas in the reference controlled case, $v_{\max } \approx 0.016$ and $\left.v_{w \max } \approx 0.014\right)$. In fact, eventually, at $t=40$ the $v_{w}$ patches do not correspond anymore to the regions of large wall-normal velocity perturbation (see figure $14(\mathrm{~b}))$. At such a time, the effect of the control on the perturbation begins to be non-negligible. Comparing figure 14, for the controlled case, with figure 15, for the uncontrolled case, one can observe that at $t=20$ the perturbations are very similar, whereas they begin to show some differences at $t=40$. In particular, in the controlled case, the structures are less inclined with respect to the streamwise direction, as one can observe by comparing the $\Lambda$ white regions in figure 15 (b) with the more smooth ones in figure 14 (b). This effect is more clearly visible at larger times (compare figure 14 (c) and figure $15(\mathrm{c})$ for $t=60$ ), where the positive and negative wall-normal velocity regions lose much of their inclination with respect to the streamwise direction. It is noteworthy that the interface regions between positive and negative wall-normal velocity correspond to regions of large streamwise vorticity, as one can observe in figure 15 by comparing the green surfaces (streamwise vorticity) with the black and white regions (negative/positive wall-normal velocity perturbation). Also in the controlled case, provided in figure 16, one can notice that the interface regions between suction and blowing zones at the wall 


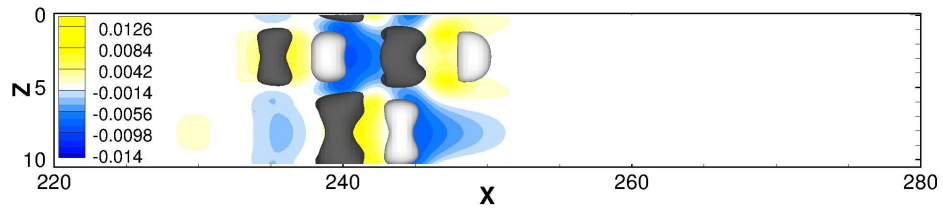

(a)

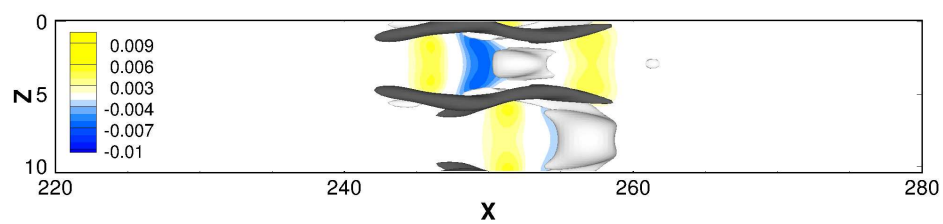

(b)

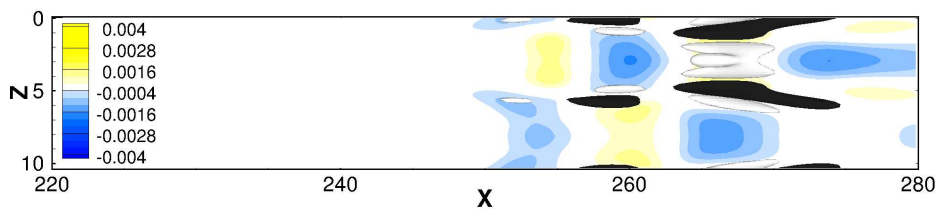

(c)

FIGURE 14. Shaded contours of the blowing and suction velocity $v_{w}$ at the wall and isosurfaces of the wall-normal velocity perturbation (white and black) at (a) $t=20\left(v^{\prime}= \pm 0.009\right)$, (b) $t=40\left(v^{\prime}= \pm 0.009\right),(\mathrm{c}) t=60\left(v^{\prime}= \pm 0.0125\right)$ for the reference case.

correspond to the location of the weaker quasi-streamwise vortices (green). By comparing figure 15 with figure 16 , it appears that the effect of the $v_{w}$ on the quasi-streamwise vortices is to weaken and stretch them in the streamwise direction, transforming pairs of strong $\Lambda$ vortices into weak, thin vortices almost aligned with the streamwise direction. This weakening and elongation of the vortices hampers the generation of the hairpin vortex, which is clearly shown at $t=100$ for the uncontrolled case in figure 17 (compare with the vorticity surfaces plotted at the same time in the reference controlled case in figure $16(\mathrm{~d}))$.

In order to understand which mechanisms induce the weakening of the vortices, we visualize the perturbation onto two $x=$ const planes for the controlled case at $t=60$ (close to the time of maximum amplitude of $v_{w}$ ). Figure 18 provides the vectors of the spanwise and wall-normal perturbation, as well as the streamwise vorticity isosurfaces (green), also identified by bold letters from A to F. The blowing and suction at the wall is represented by the thick red vectors, which have been magnified 800 times for visualization purpose. One can observe that, in both plots, the $v_{w}$ vectors tend to counterbalance the rotation of the vortices, by applying a positive (negative) velocity in the right (left) half of clockwise vortices, and viceversa for counterclockwise vortices. The effect is that the vortices are strongly weakened, since $v_{w}$ opposes their rotation. It is noteworthy that such a rotation-counterbalancing effect is different from the compensation of the wallnormal velocity observed at small times, since now the balancing effect is concentrated in the zones of streamwise vorticity, and does not lead to a cancellation of the wall-normal perturbation but to a weakening of the vortices (as shown in figure 16). Thus, as a result of the control, the vortices decrease their strength and reduce the transport of streamwise 


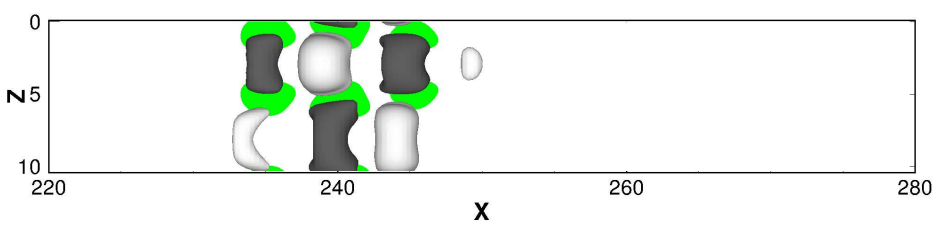

(a)

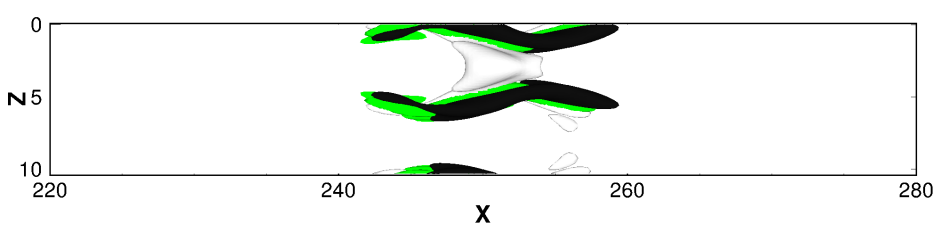

(b)

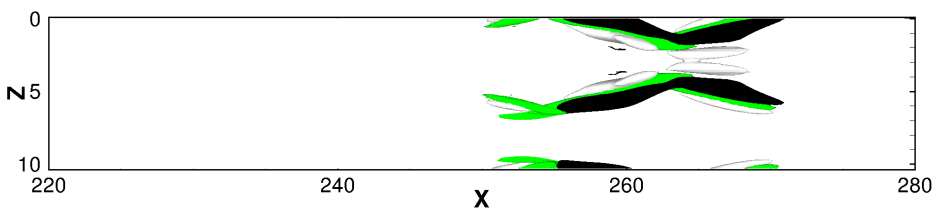

(c)

FiguRE 15. Isosurfaces of the wall-normal velocity (white and black) and of the streamwise vorticity (green) perturbation at (a) $t=20\left(v^{\prime}= \pm 0.009,\left|\omega_{x}{ }^{\prime}\right|=0.15\right)$, (b) $t=40\left(v^{\prime}= \pm 0.015\right.$, $\left.\left|\omega_{x}{ }^{\prime}\right|=0.3\right)$, (c) $t=60\left(v^{\prime}= \pm 0.035,\left|\omega_{x}{ }^{\prime}\right|=0.4\right)$ for an uncontrolled DNS initialized by the NLOP.

momentum, hampering the generation of large streamwise perturbations (streaks in the linear optimal case, $\Lambda$-shaped regions for the nonlinear case).

As discussed in Cherubini et al. (2011b), the nonlinear term which is crucial to allow the sustainment and the subsequent transition to turbulence of a finite amplitude perturbation in a boundary-layer flow, is the $w w_{z}$ term, which has been found to be intimately linked to the strength and the spanwise inclination of the vortices. When such a term is artificially switched off in a simulation, the nonlinear optimal perturbation is found to laminarize very quickly, the initial vortices being straightened up, and fading away in a very small time (see Cherubini et al. (2011b)). To understand how the nonlinear optimal control law affects such a term, we have computed the time evolution of the rms of $w w_{z}$, for the controlled and uncontrolled cases. Figure 19 shows that in the controlled case such a term begins to decrease already at $t \approx 35$, close to the time at which the rotation-counterbalancing effect appears. At target time $(t=T=100)$, the rms value of $w w_{z}$ in the controlled case is more than 3 orders of magnitude smaller than in the uncontrolled one, meaning that the damping of such a nonlinear term is very strong. Thus, the control law found here strongly damps such a nonlinear term, hindering the main mechanism inducing self-sustainment of the finite-amplitude perturbation. The blowing and suction at the wall also tends to straighten the vortices, decreasing their tilting angle in the wall-normal direction. Figure 20 provides the streamwise vorticity surfaces at $t=100$ for the controlled (a) and the uncontrolled (b) case, clearly showing a lower angle of the vortices in the wall-normal direction in the controlled case with respect to the uncontrolled case. This may hamper the formation of the hairpin vortex (see figure 


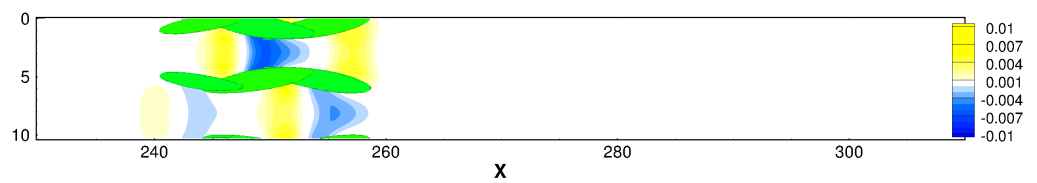

(a)

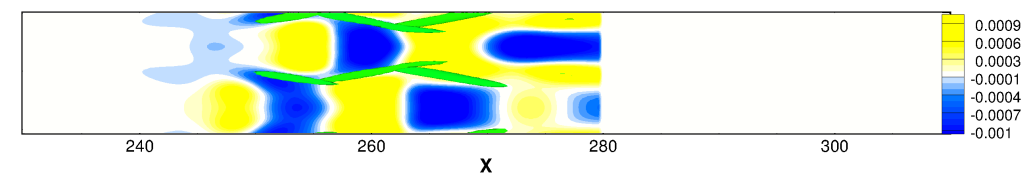

(b)

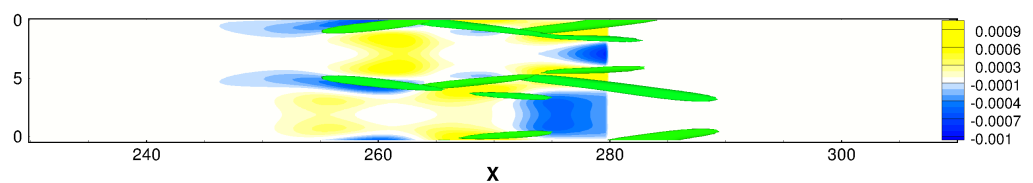

(c)

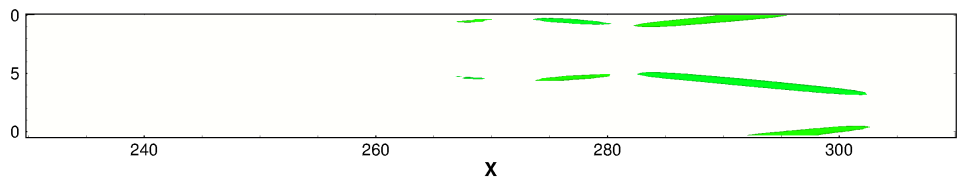

(d)

FiguRE 16. Shaded contours of the blowing and suction velocity $v_{w}$ at the wall and isosurfaces of the streamwise vorticity perturbation (green) at (a) $t=40$, (b) $t=60\left(\left|\omega_{x}{ }^{\prime}\right|=0.19\right)$, (c) $t=80$, and (d) $t=100\left(\left|\omega_{x}{ }^{\prime}\right|=0.1\right)$ for the reference case.

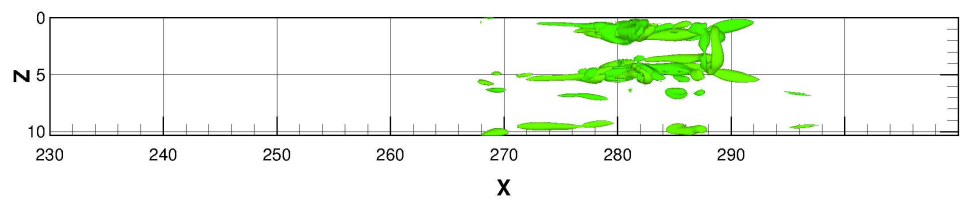

Figure 17. Isosurfaces of the $\mathrm{Q}$ criterion at $t=100$ extracted from an uncontrolled DNS initialized by the NLOP.

17 ), which is characterized by legs having an inclination close to 45 degrees (see Adrian (2007)).

It is interesting to verify that the optimal suction and blowing law found in the reference case, along with the laminarizing mechanisms analyzed above, are also found for different values of the independent parameters of the minimisation. Concerning the localization of the disturbance slot, figures 21 (a) and (b) show the blowing and suction contours at $t=5$ and $t=20$, respectively, for case $\mathrm{V}$ (blowing and suction on the second quarter of the disturbance slot), $T=100$ and $\gamma^{2}=0.2$ (solid contours). Comparing the solid contours with the shaded ones (reference case), one can notice that, except for the localization, the shape of the contours is almost the same. Similar results have been found for case VII, in which five localized disturbance strips have been placed on the second quarter 


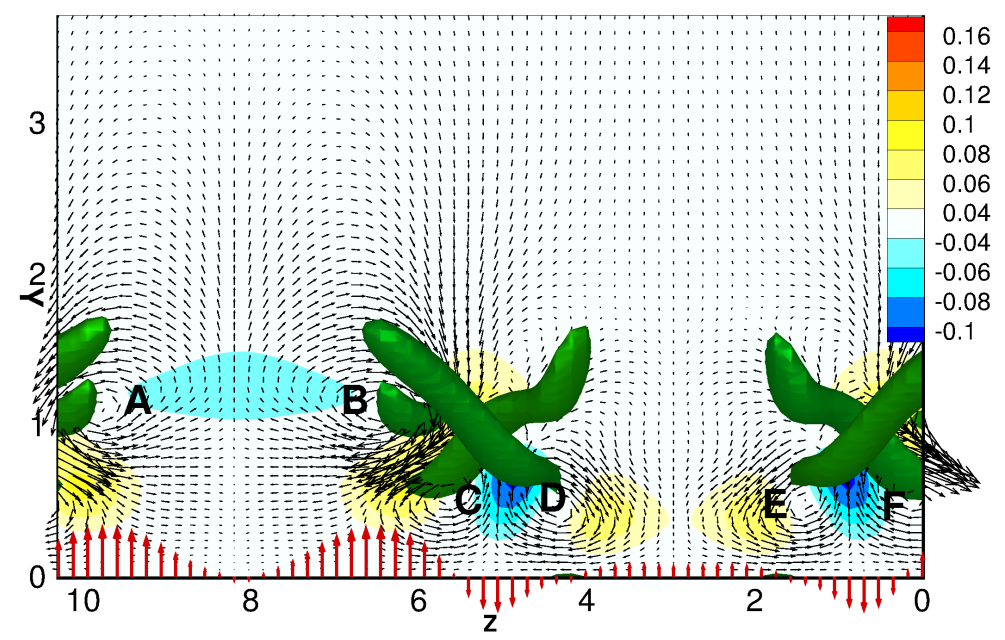

(a)

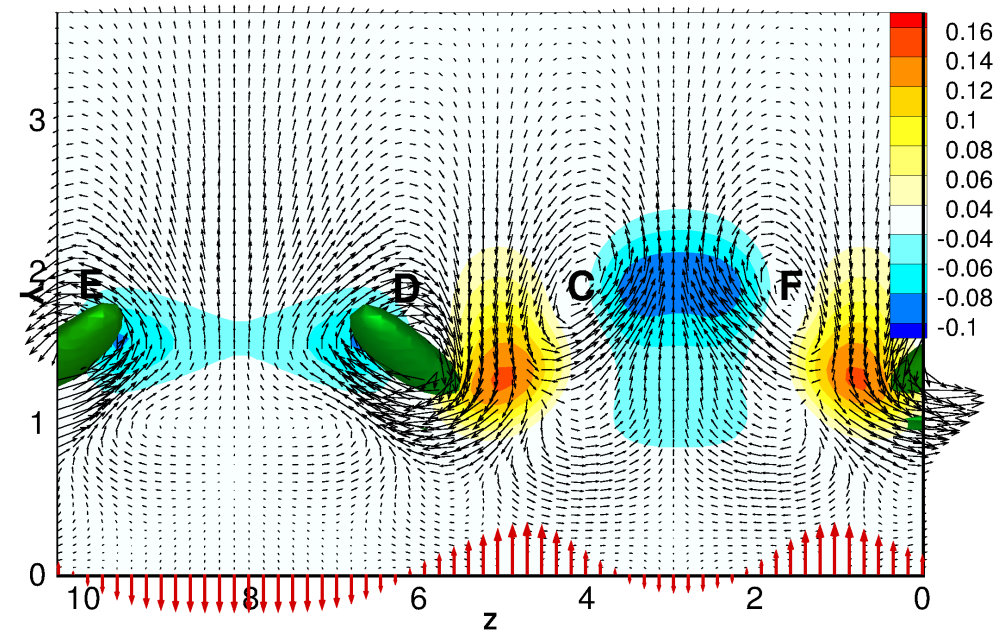

(b)

FIGURE 18. Vectors of the spanwise and wall-normal components, and shaded contours of the streamwise component of the velocity perturbation on the planes (a) $x_{s}=260$, (b) $x_{s}=270$, in the reference case, extracted at $t=60$. The green isosurfaces represent the streamwise vorticity at $x>x_{s}$. The blowing and suction velocity profiles at the wall have been magnified of 800 times and are indicated by the thick red vectors.

of the disturbance slot. Figure 22 shows that the shape of the positive and negative $v_{w}$ contours is the same also when the disturbance strips are distributed in space. The actuation zone is narrower than in the reference case, so that, to obtain the same decrease of the energy gain of the perturbation, a higher amplitude of the blowing and suction is needed. Finally, Figure 23 shows the solid contours of $v_{w}$ for case 0 with target time $T=50$. Although the shape of $v_{w}$ appears to follow that for the reference case, the amplitudes of the blowing and suction are much weaker. This is due to the fact that the 


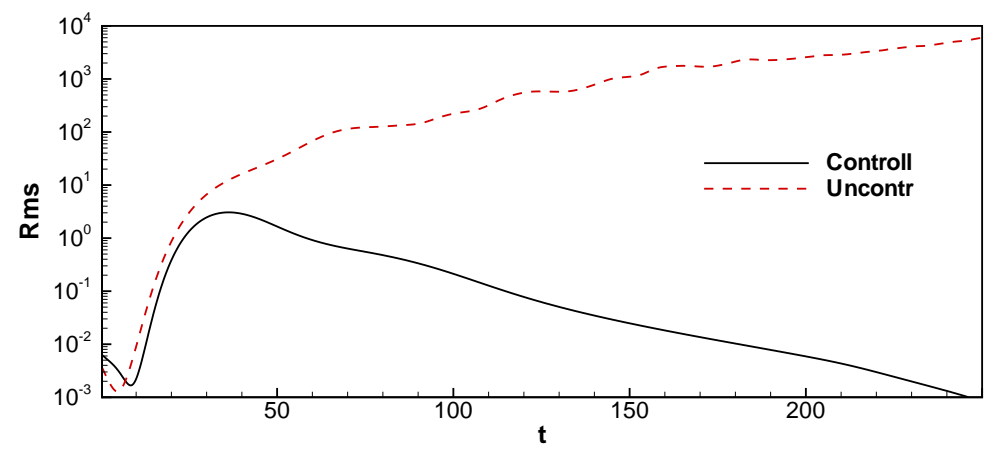

FiguRE 19. Time evolution of the rms values of the nonlinear term $w w_{z}$ in the reference controlled (solid line) and uncontrolled case (dashed line), initialized by the NLOP.

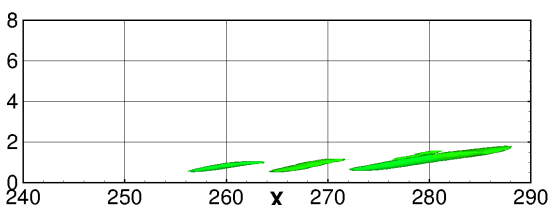

(a)

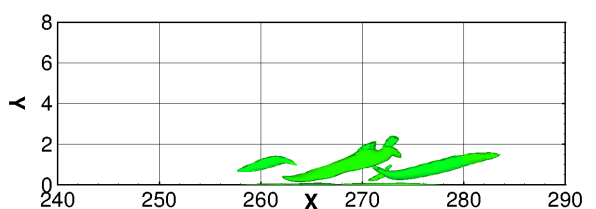

(b)

FIGURE 20. Streamwise vorticity isosurfaces at $t=100$ for (a) the reference controlled case $\left(\left|\omega_{x}^{\prime}\right|=0.12\right)$ and (b) the uncontrolled case $\left(\left|\omega_{x}^{\prime}\right|=0.4\right)$, initialized by the NLOP.

controller has not enough time to induce a large decrease of the perturbation energy, so it economizes on the control cost to minimize the value of the objective function. As a consequence, when the target time is lower than or equal to $T=50$, the laminarization cannot be observed.

Similar results have also been obtained for an initial linear optimal disturbance. In particular, even if the shape of the blowing and suction is different (as shown in figure 13 ), the regions of interface between positive and negative values of $v_{w}$ again correspond to streamwise vortices, and tend to weaken them in time. On the other hand, when a linearized control procedure is used, the interface zones between positive and negative $v_{w}$ do not correspond to high streamwise vorticity (see the contours in figure 13 (a) and compare with the uncontrolled disturbance in figure 15), since the linearized NavierStokes equations cannot predict correctly the evolution of finite amplitude perturbations. These results show that including nonlinear effects is crucial for controlling the energy growth of a finite amplitude perturbation in a boundary-layer flow.

\section{Summary}

In the present work, a full-state optimal control strategy, based on the nonlinear NavierStokes equations, is provided, aiming at hampering the rapid growth of finite-amplitude perturbations in a boundary-layer flow. To this end, a Lagrange multipliers technique has been used to find the blowing and suction control law at the wall providing the largest decrease of the energy of a given perturbation at a certain target time. The objective function of the minimisation procedure is the sum of the energy gain of the initial perturbation and the control cost, weighted by a parameter $\gamma$. Localized disturbance slots 


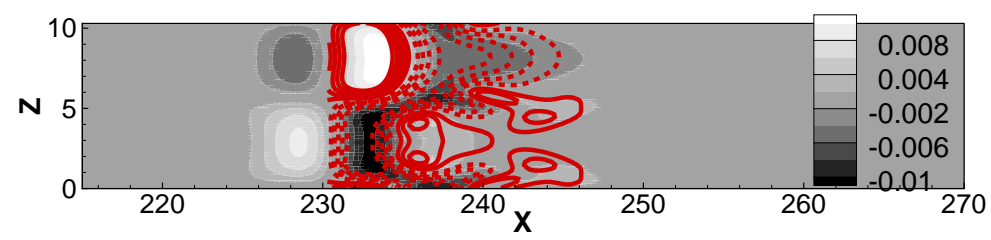

(a)

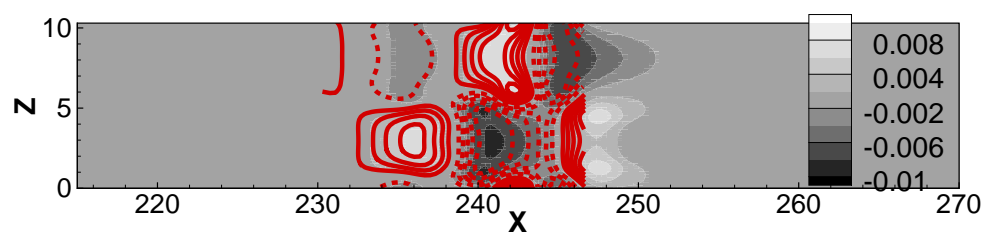

(b)

FIgURE 21. Blowing and suction at the wall at (a) $t=5$ and (b) $t=20$ for a nonlinear control in the reference case (shaded contours) and in case $\mathrm{V}$ (solid and dashed contours for positive and negative values of $v_{w}$, respectively), with $T=100, \gamma^{2}=0.2$, initialized by the NLOP.

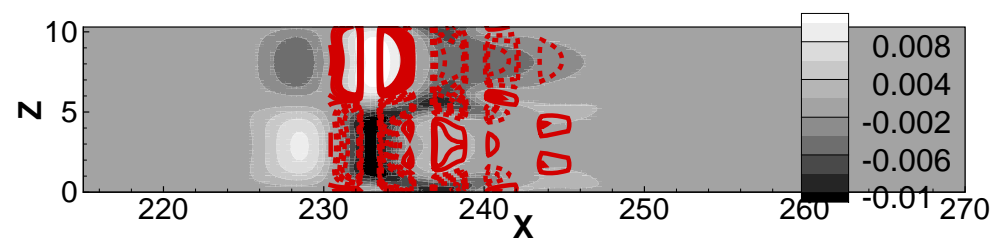

(a)

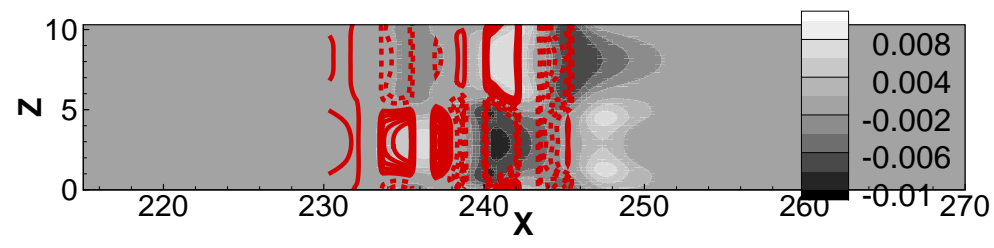

(b)

Figure 22. Blowing and suction at the wall at (a) $t=5$ and (b) $t=20$ for a nonlinear control in the reference case (shaded contours) and in case VII (solid and dashed contours for positive and negative values of the $v_{w}$, respectively), with $T=100, \gamma^{2}=0.2$, initialized by the NLOP.

of different lengths have been used to actuate the blowing and suction law. Moreover, in order to generalize the approach, two different optimally-growing finite-amplitude initial perturbations have been considered to initialize the procedure: a linear and a nonlinear optimal perturbation. They have been computed by a maximization of the perturbation energy gain in a linearized and fully nonlinear framework, respectively. Such perturbations are able to lead the flow very rapidly to transition by inducing regions with localized, convecting saturated streaks or $\Lambda$-shaped flow structures, respectively. The 


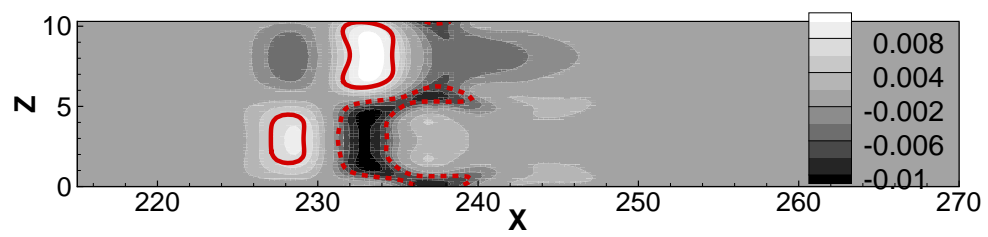

(a)

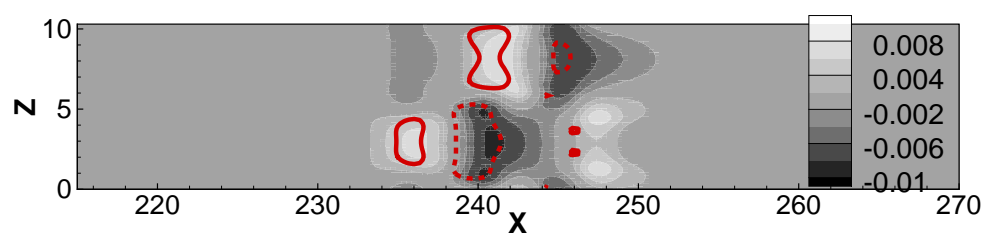

(b)

Figure 23. Blowing and suction at the wall at (a) $t=5$ and (b) $t=20$ for a nonlinear control in the reference case (shaded contours) and for a control obtained with $T=50$ (solid and dashed contours for positive and negative values of the $v_{w}$, respectively), $\gamma^{2}=0.2$, case 0 , initialized by the NLOP.

proposed nonlinear control procedure is capable of leading such perturbations back to the laminar fixed point, provided that the target time of the minimisation and the region in which the blowing and suction is applied are chosen to be large enough. In order to assess the effect of the nonlinear terms on the damping of the disturbance energy gain, an equivalent control procedure based on the linearized Navier-Stokes equations has been also performed for the same set of parameters. Such a linear optimal control has been found much less effective, not being able to lead the finite-amplitude perturbations to laminarization. Moreover, the dependence of the perturbation energy damping on the localization and length of the disturbance slot, has been analyzed. Provided that a suitable position of the disturbance slot is chosen with respect to the perturbation, even when the actuator strip is strongly localized, a remarkable reduction of the energy gain is obtained. This suitable position can be identified as the high-sensitivity region of the time-evolving perturbation to blowing and suction at the wall. In the present work, the sensitivity has been evaluated by simply analysing the response of the control for seven positions of the control strip at the wall. In a real flow configuration, a suitable sensor should trigger the start of the control actuation in order to synchronize the control law with the perturbation.

The result of the control procedure is a blowing and suction law consisting of alternated patches of wall-normal velocity perturbation, which initially show a rounded shape closely following the flow regions of finite wall-normal perturbation, but with opposite sign. Thus, at early times the blowing and suction law appears to damp the velocity perturbation by compensating its wall-normal component by applying a velocity perturbation of opposite sign at the wall. However, at larger times $(t>40)$ the blowing and suction structures change into $\Lambda$-shaped patches of alternated wall-normal velocity perturbation, following the zones of large streamwise vorticity. In particular, the blowing and suction law appears to affect the vortices by weakening their amplitude and reducing their inclination, by counterbalancing their rotation applying a flow rotation of different 
sign at the wall. Thus, the nonlinear control law appears to affect the finite amplitude perturbation by means of two distinct mechanisms: i) a wall-normal velocity-compensation at small times; ii) a rotation-counterbalancing effect at larger times. These two effects are observed also when varying the independent parameters of the minimisation (the target time, the cost parameter, the streamwise extents of disturbance slot) so they appear to be a general effective feature of the control and might be used also in other control strategies using different approaches. On the other hand, when the nonlinear effects are neglected, the linear optimal blowing and suction law shows a very different shape, consisting of elongated regions of wall-normal perturbation velocity, which are not able to hamper the growth of the nonlinearly growing perturbations. Thus, it appears that including the nonlinear effects into a model for determining the control law is crucial to effectively damp the growth of finite-amplitude perturbations in the boundary-layer flow.

The present nonlinear control strategy has been found very effective to control the growth of finite-amplitude perturbations in the boundary-layer flow. However, it cannot be considered as a practical solution to control a real flow, being very high-dimensional, and assuming the knowledge of the full state of the system at the initial time. Future works will aim at extending the current nonlinear full-state control strategy to take into account the presence of sensors for flow estimation (for instance, using the model predictive control), and/or using a model to reduce the dimensions of the system.

\section{Acknowledgments}

Some computations have been performed on the Power 6 of the IDRIS, France.

\section{REFERENCES}

Adrian, R. J. 2007 Hairpin vortex organization in wall turbulence . Phys. Fluids 19, 041301.

Alizard, F., Cherubini, S. \& Robinet, J. Ch. 2009 Sensitivity and optimal forcing response in separated boundary layer flows. Physics of Fluids 21, 064108.

Bagheri, S. \& Henningson, D. H. 2011 Transition delay using control theory. Phil. Trans. R. Soc. 369, 1365-1381.

Barbagallo, A., Dergham, G., Sipp, D., Schmid, P. J. \& Robinet, J. C. 2012 Closed-loop control of unsteadiness over a rounded backward-facing step. J. Fluid Mech. 703, 326-362.

Bewley, T. R. \& LiU, S. 1998 Optimal and robust control and estimation of linear paths to transition. J. Fluid Mech. 365, 305-349.

Bewley, T. R., Moin, P. \& Temam, R. 2001 Dns-based predictive control of turbulence: an optimal benchmark for feedback algorithms flows. J. Fluid Mech. 447, 179-225.

Bottaro, A. 1990 Note on open boundary conditions for elliptic flows . Num. Heat Transfer B 18, 243-256.

Brandt, L., Schlatter, P. \& Henningson, D. S. 2004 Transition in a boundary layers subject to free-stream turbulence. J. Fluid Mech. 517, 167-198.

Cherubini, S. \& De Palma, P. 2012 Nonlinear optimal perturbations in a Couette flow: bursting and transition. J. Fluid Mech. 716, 251-279.

Cherubini, S., De Palma, P., Robinet, J.-Ch. \& Bottaro, A. $2010 a$ Rapid path to transition via nonlinear localized optimal perturbations . Phys. Rev. E 82, 066302.

Cherubini, S., De Palma, P., Robinet, J.-Ch. \& Bottaro, A. $2011 a$ Edge states in a boundary layer . Phys. of Fluids 23, 051705.

Cherubini, S., De Palma, P., Robinet, J.-Ch. \& Bottaro, A. $2011 b$ The minimal seed of turbulence transition in a boundary layer . J. Fluid Mech. 689, 221-253.

Cherubini, S., Robinet, J.-Ch., Bottaro, A. \& De Palma, P. $2010 b$ Optimal wave packets in a boundary layer and initial phases of a turbulent spot . J. Fluid Mech. 656, 231-259.

Chevalier, M., Hoepffner, J., Akervik, E. \& Henningson, D. S. 2007 Linear feedback 
control and estimation applied to instabilities in spatially developing boundary layers. $J$. Fluid Mech. 588, 163-187.

Chevalier, M., Hogberg, M., Berggren, M. \& Henningson, D. S. 2002 Linear and nonlinear optimal control in spatial boundary layers. AIAA-2012-2755 .

Choi, H., Jeon, W.P. \& Kim, J. 2008 Control of flow over a bluff body. J. Fluid Mech. 40, $113-139$.

Duguet, Y., Schlatter, P., Henningson, D. S. \& Eckhardt, B. 2012 Self-sustained localized structures in a boundary-layer flow . Phys. Rev. Lett. 108, 044501.

Eckhardt, B., Schneider, T. M., Hof, B. \& Westerweel, J. 2007 Turbulence transition of pipe flow . Annu. Rev. Fluid Mech. 39, 447-468.

Fransson, J.H.M., Talamelli, A., Brandt, L. \& Cossu, C. 2006 Delaying transition to turbulence by a passive mechanism . Phys. Rev. Lett. 96 (6), 064501.

Giannetti, F. \& Luchini, P. 2007 Structural sensitivity of the first instability of the cylinder wake. J. Fluid Mech. 581, 167-197.

Hammond, E.P., Bewley, T. R. \& Moin, P. 1998 Observed mechanisms for turbulence attenuation and enhancement in opposition-controlled wall-bounded flows . Phys. Fluids 10, 2421-2423.

Herve, A., Sipp, D., Schmid, P. J. \& Samuelides, M. 2012 A physics-based approach to flow control using system identification. J. Fluid Mech. 702, 26-58.

Hof, B., van Doorne, C.W.H., Westerweel, J., Nieuwstadt, F.T.M., Faisst, H., Eckhardt, B., Wedin, H., Kerswell, R.R. \& Waleffe, F. 2004 Experimental Observation of Nonlinear Traveling Waves in Turbulent Pipe Flow . Science 305, 1594-1598.

Huerre, P. \& Rossi, M. 1998 Hydrodynamic instabilities in open flows. . Hydrodynamics and Nonlinear Instabilities .

Kim, J. \& Bewley, T. R. 2007 A linear systems approach to flow control. Ann. Rev. Fluid Mech. 39, 383-417.

Lewis, F. L. \& Syrmos, L. V. 1995 Optimal control .

Marquet, O., Sipp, D., Chomaz, J.-M. \& Jacquin, L. 2008 a Amplifier and resonator dynamics of a low-Reynolds-number recirculation bubble in a global framework. J. Fluid Mech. 605, 429-443.

MARquet, O., SiPP, D. \& JACQUin, L. $2008 b$ Sensitivity analysis and passive control of cylinder flow. J. Fluid Mech. 615, 221-252.

Monokrousos, A., Bottaro, A., Brandt, L., Di Vita, A. \& Henningson, D. S. 2011 Nonequilibrium thermodynamics and the optimal path to turbulence in shear flows. Phys. Rev. Lett. 106, 134502.

NAGATA, M. 1990 Three-dimensional finite amplitude solutions in plane Couette flow . J. Fluid Mech. 217, 519-527.

Passaggia, P.Y. \& Ehrenstein, U. 2013 Adjoint based optimization and control of a separated boundary-layer flow. Eur. J. Mech. B/Fluids in press.

Pringle, C. C. T. \& Kerswell, R.R. 2010 Using nonlinear transient growth to construct the minimal seed for shear flow turbulence. Phys. Rev. Lett. 105, 154502.

Pringle, C. C. T., Willis, A. P. \& Kerswell, R.R. 2012 Minimal seeds for shear flow turbulence: using nonlinear transient growth to touch the edge of chaos. J. Fluid Mech. 702, 415-443.

Rabin, S. M. E., Caulfield, C. P. \& Kerswell, R.R. 2012 Variational identification of minimal seeds to trigger transition in plane Couette flow . J. Fluid Mech. 712, 244-272.

Schmid, P. \& Henningson, D. 2001 Stability and transition in shear fows. Springer.

Schneider, T. M., Eckhardt, B. \& Yorke, J.A. 2007 Turbulence Transition and the Edge of Chaos in Pipe Flow . Phys. Rev. Lett. 99, 034502.

Schoppa, W. \& Hussain, F. 2002 Coherent structure generation in near-wall turbulence. $J$. Fluid Mech. 453, 57-108.

Semeraro, O., Bagheri, S., Brandt, L. \& Henningson, D. S. 2011 Feedback control of three-dimensional optimal disturbances using reduced-order models. J. Fluid Mech. 677, $63-102$.

Shahinfar, S., Sattarzadeh, S. S., Fransson, J. H. M. \& Talamelli, A. 2012 Revival of classical vortex generators now for transition delay. Phys. Rev. Lett. 109, 074501. 
Skufca, J. D., Yorke, J.A. \& Eckhardt, B. 2006 Edge of chaos in a parallel shear flow . Phys. Rev. Lett. 96, 174101.

Strykowski, P. J. \& Sreenivasan, K. R. 1990 On the formation and suppression of vortex "shedding" at low reynolds numbers . J. Fluid Mech. 218, 71-107.

Verzicco, R. \& Orlandi, P. 1996 A finite-difference scheme for the three-dimensional incompressible flows in cylindrical coordinates . J. Comp. Phys. 123 (2), 402-414.

Viswanath, D. \& Cvitanovic, P. 2009 Stable manifolds and the transition to turbulence in pipe flow . J. Fluid Mech. 627, 215.

Waleffe, F. 1997 On a self-sustaining process in shear flows . Phys. Fluids 9, 883-900.

Waleffe, F. 1998 Three-dimensional states in plane shear flow . Phys. Rev. Lett. 81, 4140-4143.

Wedin, H. \& Kerswell, R.R. 2004 Exact coherent structures in pipe flow: travelling wave solutions . J. Fluid Mech. 508, 333-371.

Zuccher, S., Luchini, P. \& Bottaro, A. 2004 Algebraic growth in a blasius boundary layer: optimal and robust control by mean suction in the nonlinear regime. Eur. J. Mech. B/Fluids 513, 135-160. 